\title{
An Efficient Approach to Obtaining Large Numbers of Distant Supernova Host Galaxy Redshifts
}

\author{
C. Lidman ${ }^{1,2,17}$, V. Ruhlmann-Kleider ${ }^{3}$, M. Sullivan ${ }^{4}$, J. Myzska ${ }^{1,5}$, P. Dobbie ${ }^{1}$, K. Glazebrook ${ }^{2,6}$, \\ J. Mould ${ }^{2,6}$, P. Astier ${ }^{7}$, C. Balland ${ }^{7,8}$, M. Betoule ${ }^{7}$, R. Carlberg ${ }^{9}$, A. Conley ${ }^{10}$, D. Fouchez ${ }^{11}$, J. Guy ${ }^{7}$, \\ D. Hardin 7 , I. Hook ${ }^{4,12}$, D. A. Howell ${ }^{13,14}$, R. Pain ${ }^{2,7}$, N. Palanque-Delabrouille ${ }^{3}$, K. Perrett ${ }^{9,15}$, \\ C. Pritchet ${ }^{16}$, N. Regnault ${ }^{7}$ and J. Rich ${ }^{3}$ \\ ${ }^{1}$ Australian Astronomical Observatory, PO Box 296, Epping NSW 1710, Australia \\ ${ }^{2}$ ARC Centre of Excellence for All-sky Astrophysics (CAASTRO), Redfern, NSW 2016, Australia \\ ${ }^{3} \mathrm{DSM} / \mathrm{IRFU} / \mathrm{SPP}, \mathrm{CEA}-\mathrm{Saclay}, \mathrm{F}-91191$ Gif-sur-Yvette, France \\ ${ }^{4}$ Department of Physics (Astrophysics), University of Oxford, DWB, Keble Road, Oxford OX1 3RH, UK \\ ${ }^{5}$ Villanova University, 800 East Lancaster Avenue Villanova, PA 19085, USA \\ ${ }^{6}$ Centre for Astrophysics and Supercomputing, Swinburne University of Technology, PO Box 218, Hawthorn, VIC 3122, Australia \\ ${ }^{7}$ LPNHE, Universite Pierre et Marie Curie Paris 6, Universite Paris Diderot Paris 7, CNRS-IN2P3, 4 place Jussieu, 75005 Paris, France \\ ${ }^{8}$ Universite Paris 11, Orsay, F-91405, France \\ ${ }^{9}$ Department of Astronomy and Astrophysics, University of Toronto, 50 St. George Street, Toronto, ON M5S 3H4, Canada \\ ${ }^{10}$ Center for Astrophysics and Space Astronomy, University of Colorado, 593 UCB, Boulder, CO 80309, USA \\ ${ }^{11}$ CPPM, CNRS-IN2P3 and University Aix Marseille II, Case 907, 13288 Marseille Cedex 9, France \\ ${ }^{12}$ INAF, Osservatorio Astronomico di Roma, via Frascati 33, 00040 Monteporzio (RM), Italy \\ ${ }^{13}$ Las Cumbres Observatory Global Telescope Network, 6740 Cortona Dr., Suite 102, Goleta, CA 93117, USA \\ ${ }^{14}$ Department of Physics, University of California, Santa Barbara, Broida Hall, Mail Code 9530, Santa Barbara, CA 93106, USA \\ ${ }^{15}$ DRDC Ottawa, 3701 Carling Avenue, Ottawa, ON, K1A 0Z4, Canada \\ ${ }^{16}$ Department of Physics and Astronomy, University of Victoria, PO Box 3055, Stn CSC, Victoria, BC V8W 3P6, Canada \\ ${ }^{17}$ Email: clidman@aao.gov.au
}

(Received January 9, 2012; Accepted May 6, 2012; Online Publication January 18, 2013)

\begin{abstract}
We use the wide-field capabilities of the 2 degree field fibre positioner and the AAOmega spectrograph on the AngloAustralian Telescope (AAT) to obtain redshifts of galaxies that hosted supernovae during the first 3 years of the Supernova Legacy Survey (SNLS). With exposure times ranging from 10 to $60 \mathrm{ks}$ per galaxy, we were able to obtain redshifts for 400 host galaxies in two SNLS fields, thereby substantially increasing the total number of SNLS supernovae with host galaxy redshifts. The median redshift of the galaxies in our sample that hosted photometrically classified Type Ia supernovae (SNe Ia) is $z \sim 0.77$, which is $25 \%$ higher than the median redshift of spectroscopically confirmed SNe Ia in the 3-year sample of the SNLS. Our results demonstrate that one can use wide-field fibre-fed multi-object spectrographs on 4-m telescopes to efficiently obtain redshifts for large numbers of supernova host galaxies over the large areas of the sky that will be covered by future high-redshift supernova surveys, such as the Dark Energy Survey.
\end{abstract}

Keywords: cosmology: observations, galaxies: clusters: general, galaxies: general, supernovae: general, white dwarfs

\section{INTRODUCTION}

The landmark discovery of the accelerating Universe more than a dozen years ago (Riess et al. 1998; Perlmutter et al. 1999) was made from observations of a few dozen distant Type Ia supernovae (SNe Ia). Since then, SN Ia samples have grown in size by an order of magnitude. Currently, the largest published individual sample of distant SNe Ia is the 3-year sample from the Supernova Legacy Survey (SNLS), which contains 252 SNe Ia extending out $z=1.06$ (Guy et al.
2010). The Dark Energy Survey (DES), which will discover $\sim 4000$ distant SNe Ia (Bernstein et al. 2011), is yet another order of magnitude larger.

All of the SNe Ia in the 3-year SNLS sample were spectroscopically confirmed. To confirm a SN Ia at $z \sim 0.7$ from its spectral features (and to obtain its redshift) typically takes about an hour of exposure time with an efficient spectrograph on an 8-10 m class telescope. These SNe are observed one at a time, as it is, at any instant, very rare to have more than one SN Ia within 1 week of maximum light visible within the 
fields of view that are available with the current generation of spectrographs on 8-10 m class telescopes. Assuming 100\% efficiency, DES will need around $4000 \mathrm{~h}$ of 8-10 m class telescope time with current instrumentation to spectroscopically confirm the $4000 \mathrm{SNe}$ Ia that they will find. Clearly, this constitutes a very large request, so alternative approaches should be investigated first.

A promising yet unproven alternative is to classify $\mathrm{SNe}$ Ia using multi-colour light curves and to obtain the redshifts from host galaxies after the SNe have faded from view. The principal advantages of this alternative are that (i) host galaxy redshifts can be obtained at any time and (ii) one can observe several hosts simultaneously if the number density of host galaxies is sufficiently high enough.

In this paper, we describe how we used the $2 \mathrm{dF}$ fibre positioner and the AAOmega spectrograph on the AngloAustralia Telescope (AAT) to obtain redshifts of galaxies that hosted supernovae in two of the four SNLS fields. A second paper reporting the results from a third SNLS field (the fourth field is not visible from the AAT) and the analysis of the three fields combined will be written once observations of that field have been taken. The current paper is divided as follows. In Section 2.1, we briefly describe the characteristics of the SNLS and explain how targets from the SNLS were selected and prioritised for spectroscopy. Then, in Sections 3 and 4, we describe the observations and outline the steps used to process the data. In the following two sections, we describe our results (Section 5) and discuss (Section 6) how our observing strategy compares with an observing strategy in which transients are observed in real time. In the final section, we summarise our main results. Unless noted otherwise, we use $\mathrm{AB}$ magnitudes throughout this paper.

\section{SNLS HOST GALAXIES}

The SNLS consisted of a rolling search of four one-square degree fields, labelled D1-D4, using the MegaCam camera (Boulade et al. 2003) on the Canada-France-Hawaii Telescope $^{1}(\mathrm{CFHT})$. During dark and grey time and over a period of 5-7 consecutive lunations, the fields were imaged every three to four nights in $g_{\mathrm{M}}, r_{\mathrm{M}}, i_{\mathrm{M}}$, and $z_{\mathrm{M}}$ filters. The survey ran for 5 years, starting in 2003 and ending in 2008. Additional details on the survey can be found in Astier et al. (2006), Sullivan et al. (2006a), and Perrett et al. (2010).

During the course of the SNLS, transient events were detected, classified, and prioritised in real time (Sullivan et al. 2006a; Perrett et al. 2010). The highest priority SN Ia candidates were then sent to telescopes at the Keck, Paranal, and Gemini Observatories for spectroscopic typing and redshifting. The spectroscopic follow-up of real-time transients is described in Howell et al. (2005), Bronder et al. (2008), Ellis et al. (2008), Balland et al. (2009), Walker et al. (2011), and H. Fahkouri et al. (in preparation).

\footnotetext{
${ }^{1}$ The SNLS fields were common to the deep synoptic survey of the CFHT Legacy Survey—http://www.cfht.hawaii.edu/Science/CFHLS/.
}

Likely SNe Ia were prioritised according to several criteria, such as, among others, the quality of the light curve fit, the measured stretch, peak apparent brightness and lightcurve phase, and the ratio of the likely peak flux of the SN Ia with respect to the flux of the host (Perrett et al. 2010). For example, SNe Ia that were likely to have a peak observerframe $i$-band magnitude fainter than $i_{\mathrm{AB}}=24.4$ were not sent for spectroscopy, since these $\mathrm{SNe}$ were likely to be beyond $z=0.9$, are difficult to confirm spectroscopically, and generally have one well-measured colour at most. The selection criteria are not treated in isolation. For example, candidates in the magnitude range of $23.8<i_{\mathrm{AB}}<24.4$ were only followed if the flux of the candidate was as large as the host. Due to the vagaries of telescope scheduling and weather, not all high-priority targets could be observed, and on some classically scheduled nights, lower priority targets were followed once the higher priority targets had been observed.

The spectroscopically confirmed sample is about $60 \%$ complete up to $z \sim 0.6$ (Perrett et al. 2010). At this redshift, the incompleteness is mostly due to the selection criteria that are applied to the light-curve data. Completeness then falls steadily as redshift increases. By $z \sim 1$, the completeness drops to $20 \%$ and then to zero by $z \sim 1.2$. The most distant spectroscopically confirmed SNe Ia in the 3-year SNLS sample is at $z=1.06$ (Guy et al. 2010).

Therefore, there are as many SNe Ia detected in the SNLS that lack spectroscopy as there are $\mathrm{SNe}$ Ia that were spectroscopically confirmed. Furthermore, most of the un-targeted $\mathrm{SNe}$ Ia will be at higher redshifts. These SNe Ia are now far too faint to observe spectroscopically, so they can no longer be classified in this way; however, with full light curves, many of them can be classified photometrically (Bazin et al. 2011). Together with a precise measure of the redshift, which can be obtained from the follow-up spectroscopy of the host, these SNe Ia can be added to the Hubble diagram and used to constrain the expansion history of the Universe, provided that the light curves are of sufficient quality and only after biases specific to both the photometric selection of the $\mathrm{SNe}$ Ia and the spectroscopic follow-up of the hosts are modelled and correctly accounted for.

Besides SNe Ia, the SNLS also contains a couple of hundred core-collapse supernovae (CC SNe) below $z=0.4$. Since CC SNe were rarely followed spectroscopically, only a fraction of these (42 in the first 3 years of the SNLS) were spectroscopically confirmed. As shown in Bazin et al. (2009), photometric classification allows one to identify CC $\mathrm{SNe}$ and, together with a measure of the redshift, the $\mathrm{CC} \mathrm{SNe}$ rate. Precise host galaxy redshifts can reduce the uncertainty of this measurement significantly.

\subsection{Target Selection}

Bazin et al. (2011) reanalysed the first 3 years of the SNLS survey and selected candidate supernovae based on the full 3year light curves. Their catalogue contains 1483 candidates, of which 1233 were matched to a galaxy with a reliable 
Table 1. Target Selection Summary

\begin{tabular}{|c|c|c|c|c|c|}
\hline Field & Target Type $^{a}$ & Priority ${ }^{b}$ & $\begin{array}{c}\text { Number of } \\
\text { Potential Targets }\end{array}$ & $\begin{array}{l}\text { Number } \\
\text { Targeted }\end{array}$ & $\begin{array}{l}\text { Completeness } \\
\text { Fraction }^{c}\end{array}$ \\
\hline D1 & White dwarfs & 6 & 7 & 7 & $\ldots$ \\
\hline D1 & SNe Ia without $z_{\mathrm{s}}$ & 5 & 70 & 69 & 45 \\
\hline D1 & $\mathrm{CC}$ without $z_{\mathrm{s}}$ & 4 & 18 & 17 & 94 \\
\hline D1 & $\mathrm{SNe}$ with a host but no $z_{\mathrm{s}}$ & 3 & 218 & 201 & 52 \\
\hline D1 & SNe without hosts and $z$ & 2 & 21 & 18 & 6 \\
\hline D1 & $\mathrm{SNe}$ with $z_{\mathrm{s}}$ & 1 & 117 & 49 & 53 \\
\hline D1 & Cluster galaxies & $1-5$ & 13 & 7 & 54 \\
\hline D4 & White dwarfs & 6 & 7 & 7 & $\ldots$ \\
\hline D4 & SNe Ia without $z_{\mathrm{s}}$ & 5 & 65 & 64 & 77 \\
\hline D4 & $\mathrm{CC}$ without $z_{\mathrm{s}}$ & 4 & 11 & 10 & 60 \\
\hline D4 & $\mathrm{SNe}$ with a host but no $z_{\mathrm{s}}$ & 3 & 129 & 125 & 60 \\
\hline D4 & SNe without hosts and $z$ & 2 & 32 & 30 & 13 \\
\hline D4 & SNe with $z_{\mathrm{s}}$ & 1 & 125 & 115 & 76 \\
\hline D1 and D4 & All SNe & $1-5$ & 806 & 698 & 57 \\
\hline
\end{tabular}

${ }^{a}$ Note that $z_{\mathrm{s}}$ represents the spectroscopic redshift: $z$ is a redshift of any kind, i.e. spectroscopic or photometric.

${ }^{b}$ The priority goes from 6 (highest) to 1 (lowest).

${ }^{c}$ The completeness fraction is the number of objects with a redshift quality flag of 3,4 , or 5 divided by the number of objects that were targeted with AAOmega.

photometric redshift (see Bazin et al. 2011 for details). Of these 1233 candidates, 485 were photometrically classified as SN Ia. In total, 176 of the 485 photometrically classified SN Ia were confirmed spectroscopically. None of the photometrically classified SNe Ia were confirmed to be supernovae of other types or Active Galactic Nuclei; however, not all 485 candidates were targeted for spectroscopy during the real-time follow-up.

For the follow-up spectroscopy with AAOmega, we split the catalogue of 1483 candidate supernovae into five categories and assign a priority to each category. Priority 5the highest priority we assign to photometrically identified $\mathrm{SNe}$ - goes to photometrically classified $\mathrm{SNe}$ Ia that lack a spectroscopic redshift. Then, in order of decreasing priority were photometrically classified $\mathrm{CC} \mathrm{SNe}$ without a spectroscopic redshift, candidate supernovae of unknown type with an assigned host but without a spectroscopic redshift (most objects in this priority have a reliable photometric redshift), candidate supernovae of unknown type without an assigned host and without a redshift of any kind (photometric or spectroscopic) and, finally, the lowest priority (priority 1), candidate supernovae of any kind with a spectroscopic redshift. If the identity of the host was unclear, the fibre was placed at the location of the supernova. Otherwise, it was placed on the nearest host.

In addition to $\mathrm{SN}$ hosts, we targeted white dwarfs from Limboz et al. (2008) and from our own search of the SNLS fields, and in the 2-h field only, galaxies in X-ray-selected clusters from the $X M M$-Large Scale Structure survey (Pierre et al. 2006). Both kinds of targets are interesting for a number of reasons related to cosmology. Hot DA white dwarfs are often used as flux calibrators. If a sufficient number of hot DA white dwarfs can be found within the supernova search fields, then an alternative and perhaps more precise method of calibrating $\mathrm{SNe}$ Ia fluxes would become available. These objects were given the highest priority (priority six) as we wanted to make sure that they were all allocated a fibre by the automated fibre allocation software (Miszalski et al. 2006). At the other end of the mass scale, the number density and clustering of galaxy clusters and the evolution of these properties with redshift depend on the properties of dark energy and can therefore be used to constrain it. The number of targets in each category and their priorities are listed in Table 1.

\subsection{Fibre Allocation}

The $2 \mathrm{dF}$ fibre positioner positions fibres over a 2-deg diameter field. The absolute minimum separation between fibres is 30 arcsec and is set by the rectangular shape of the magnets that hold the fibre buttons to the metallic field plate. If the separation between a given pair of supernova hosts is smaller than the minimum permitted fibre separation, then both hosts cannot be observed at the same time. The allocation of fibres to targets is then done on the basis of this requirement, the number of available fibres and target priorities (Miszalski et al. 2006).

From 3 years of the SNLS data, the number of supernovae per SNLS field is slightly larger than the number of fibres that are available with $2 \mathrm{dF}$. Each SNLS field has a field of view (FoV) of $1 \mathrm{deg}^{2}$, so there is the additional constraint that the fibres need to be placed within an area that is about a factor of 3 smaller than the full field available with $2 \mathrm{dF}$. It is interesting to see what fraction of the fibres were allocated to supernova hosts. A fraction that is significantly smaller than $100 \%$ is an indication of significant clustering.

As an example, we compute the fraction of fibres that were assigned to supernova host galaxies for the D4 field, which has the smallest difference between the number of targets and the number of fibres. The numbers listed in Table 1 cannot 
be used to compute this fraction directly because multiple configurations were used for the D4 field. Instead, we simply examine the fraction of fibres that were assigned in each configuration individually and average the results.

For the D4 field, there were 362 supernovae of all priorities. Out of 339 fibres available for plate 1 and 332 fibres available for plate $0^{2}$, respectively, 332 and 325 of them were allocated to supernova hosts. The remaining fibres were allocated to white dwarfs, which had higher priority than the supernova hosts. The allocation rate of fibres to supernova hosts was above $95 \%$ and would have been $100 \%$ if only the supernova hosts were targeted.

Interestingly, Carlberg et al. (2008) find that SNe Ia are clustered more strongly than the average galaxy. A similar study for CC SNe has not been done. While there certainly were cases where two host galaxies could not be observed simultaneously because they were too close to each other, the supernova catalogue was large enough to ensure that all fibres could be used efficiently.

The field covered by the DES camera (Flaugher et al. 2010) is three times larger than MegaCam on CFHT, the camera used by the SNLS, and is similar to the field covered by $2 \mathrm{dF}$. If the number of supernova discoveries per season and per unit area is similar for DES and SNLS, then it would take only 1 year of searching with the DES camera to find as many supernova per pointing as there are $2 \mathrm{dF}$ fibres. These supernova will be spread over the entire $2 \mathrm{dF}$ field, so the chance of fibre collisions will be smaller than it was for the follow-up of SNLS host galaxies.

\section{OBSERVATIONS}

Fibres from $2 \mathrm{dF}$ feed AAOmega (Smith et al. 2004), which is a bench-mounted double-beam spectrograph that is located in one of the Coudé rooms at the base of the AAT. The red and blue arms of the spectrograph are split by a dichroic. In the blue arm, we used the $580 \mathrm{~V}$ grating, and in the red arm, we used the $385 \mathrm{R}$ grating. The combined spectra cover the wavelength interval from 3300 to $8840 \AA$ at spectral resolution of about 1400 .

The data were taken during clear conditions. The image quality, as measured by the $2 \mathrm{dF}$ focal plane imager, which can be positioned in front of the observing plate but behind the $2 \mathrm{dF}$ corrector, varied between 1.1 and 1.7 arcsec. Each fibre has a diameter of approximately 2.1 arcsec. Distortions from the corrector mean that fibres near the field edge subtend a slightly larger region than fibres in the centre. The data were collected over four consecutive nights starting on 2011 August 24.

\footnotetext{
${ }^{2}$ At the time of the observations, 357 fibres were available on plate 0 and 364 fibres were available on plate 1 . After subtracting 25 fibres that were used to measure the sky background, approximately 330 fibres were used to select targets in the SNLS fields.
}

Each integration lasted $2500 \mathrm{~s}$ and a given configuration was observed between two to four times before switching to a new configuration. Immediately after a new configuration was tumbled into place, a fibre flat-field and a fibre arc were obtained.

Each SNLS field was configured for plate 0 and again for plate 1 . Since the number of fibres for each plate are different, not all objects observed in plate 0 were also observed in plate 1 and vice versa. Half way through the run, we processed the data that had been taken for the D4 field by that time, allowing us to re-prioritise objects that could be assigned a redshift from clearly identified spectral features.

All data were taken with the telescope pointing above an altitude of $40^{\circ}$. The total integration times for the D1 and D4 fields were 32500 and $60000 \mathrm{~s}$, respectively. The time spent on any given target, however, might have been less than this as it may not have been allocated a fibre in all the configurations. Less time was spent on the D1 field because of time lost to cloud during the second half of the third night and because of the smaller number of hours the D1 field was at a suitable hour angle. At the end of each night, five $2500-\mathrm{s}$ dark frames were taken.

In order to derive an estimate of the sky background, which is used in the processing of the data, 25 fibres were allocated to observe regions within the SNLS fields that were free of objects. We used version T0006 of the stacks produced by TERAPIX ${ }^{3}$ to select these regions. These stacks reach a limiting magnitude of $r_{\mathrm{AB}} \sim 26$, which is $\sim 4$ mag fainter than the median magnitude of galaxies targeted with AAOmega.

\section{DATA REDUCTION}

We used version 4.66 of the $2 \mathrm{dF}$ data reduction pipeline ${ }^{4}$ to process the data taken with AAOmega. The steps for processing data from the red and blue arms are similar although not identical. For both arms, we define the spectral trace of each fibre using the fibre flat fields, then extract spectra from the science frames, arcs, and flat fields, wavelength calibrate the extracted spectra from both the science data and the fibre flats, flat field the extracted spectra using the spectra from the fibre flats, and subtract the sky using the spectra extracted from the sky fibres.

For the blue arm, we first subtract a dark frame from the data to reduce the impact of the bad columns in the blue detector, we remove scattered light, and we do the wavelength calibration with the arc. For the red arm, we remove residuals from night sky lines using a Principle Component Analysis model for the residuals and we use night sky lines for the wavelength calibration.

The data from the different configurations are then coadded. Not all configurations were identical. For example, some targets were observed in one configuration but not another,

\footnotetext{
${ }^{3} \mathrm{http}: / /$ terapix.iap.fr/

${ }^{4}$ http://www.aao.gov.au/AAO/2df/aaomega/
} 
Table 2. Description of Redshift Flags

\begin{tabular}{ll}
\hline \hline Redshift Flag & \multicolumn{1}{c}{ Description } \\
\hline- & Not targeted with AAOmega \\
0 & Flawed spectrum \\
1 & Poor spectrum \\
2 & Possible redshift \\
3 & Probable redshift \\
4 & Secure redshift \\
5 & Secure redshift. High-quality spectrum, more than \\
& three features clearly visible \\
6 & Not extragalactic \\
\hline \hline
\end{tabular}

and some targets were observed with different fibres in different configurations. The pipeline keeps track of the objects that each fibre targets, so coadding the spectra on an objectby-object basis is straightforward. At this stage, we use our own scripts, coded in PYTHON and IRAF, ${ }^{5}$ to coadd the spectra, since given the long exposure times and the large number of exposures we needed better control of selecting which spectra went into the final sum than what is currently possible with the pipeline. The final step is to splice the blue and red halves of each spectrum.

The redshifts are measured with the Jan 2008 version of RUNZ (Drinkwater et al. 2010). The most commonly identified features in the spectra are emission lines from the [O II] $\lambda \lambda$ 3726, 3728 and [O III] $\lambda \lambda$ 4959, 5007 doublets, emission lines from $\mathrm{H}$-alpha and $\mathrm{H}$-beta, and absorption lines from higher order Balmer transitions and $\mathrm{Ca}$ II $\mathrm{H}$ and $\mathrm{K}$. In a few spectra, we can identify lines such as [Ne III] $\lambda 3869$, [N II] $\lambda$ 6549, and [N II] $\lambda 6583$, and the [S II] $\lambda \lambda 6716$, 6730 doublet. Spectra are assigned a redshift, a redshift uncertainty, and a quality flag, following the scheme outlined in Drinkwater et al. (2010) and listed in Table 2. The flag provides additional information about each spectrum, such as the level of assessment that the redshift is correct. The reliability of these quality flags is quantified in Drinkwater et al. (2010), albeit in the context of their sample of highly starforming emission line galaxies. Redshifts that have the flag set to 4 or 5 are considered to be secure, since the redshifts are obtained from at least two clearly identified features. Objects with the flag set to 3 have less secure redshifts. Usually, these spectra have one clear emission line, which we assume to be [O II]. Only spectra with flags 3 or higher are considered in this paper. In the following sections, we label objects that have a quality flag of 4 or 5 as objects with secure redshifts and label objects that have a quality flag of 3 as objects with probable redshifts.

\footnotetext{
${ }^{5}$ IRAF is distributed by the National Optical Astronomy Observatories, which are operated by the Association of Universities for Research in Astronomy, Inc., under the cooperative agreement with the National Science Foundation.
}

\section{RESULTS}

\subsection{Redshift Completeness}

The percentage of targets for which we obtained a redshift with AAOmega (the completeness fraction) is generally highest in the D4 field. The amount of time observing the D4 field was almost a factor of 2 longer than the time used to observe the D1 field, so the higher percentage is a reflection of the longer integration time.

The completeness fraction behaves similarly for the two fields. It is highest for photometrically classified CC SNe that did not have spectroscopic redshifts and lowest for supernovae that had neither a spectroscopic redshift nor a photometric one. Hence, in what follows, we pool the results of the two fields together when analysing completeness as a function of magnitude, redshift, and target type.

Histograms showing the number of galaxies with a redshift from AAOmega are shown in Figure 1. Supernovae for which the $r$-band magnitude of the host was unavailable are not shown. Not surprisingly, the median redshift of galaxies with a probable redshift (the quality flag set to 3 ) from AAOmega is higher than the median redshift of galaxies that have a secure one. Usually, the [O II] doublet is the only line that is detected in galaxies with probable redshifts. Other lines, such as the [O III] doublet, are not detected because either they have been redshifted beyond the red end of the spectral coverage (occurs by $z \sim 0.76$ ) or, less often, they are too weak. At the resolution provided by AAOmega, the [O II] doublet is only just resolved, which can sometimes help in making a redshift marked as possible (quality flag 2) to one that is probable (quality flag 3 ).

In Figure 2, we split the magnitude and redshift histograms according to the photometric classification (i.e. $\mathrm{SNe} \mathrm{Ia}, \mathrm{SNe}$ CC, or other; Bazin et al. see 2011, for details). There are a couple of things to note in these histograms.

In particular, the middle histogram in the lower plot shows that about $90 \%$ of galaxies that hosted a SNe Ia at $z \sim 0.5$ have either a secure redshift or a probable one. By $z \sim 1$, this drops to about $50 \%$, of which about half are secure. Not shown in this lower panel are supernovae that did not have either a spectroscopic redshift (from any source) or a photometric one. These make up about $15 \%$ of all supernovae that were targeted with AAOmega.

The median redshift of objects photometrically classified as $\mathrm{CC} \mathrm{SNe}$ is lower than the median redshifts of the other two classifications. Primarily, this is driven by the absolute magnitude of $\mathrm{CC} \mathrm{SNe,} \mathrm{which} \mathrm{are,} \mathrm{on} \mathrm{average,} \mathrm{about} 2 \mathrm{mag}$ fainter than $\mathrm{SNe}$ Ia. The median apparent magnitude of galaxies that hosted CC SNe is more than 1 mag brighter, leading to a relatively high completeness.

\subsection{White Dwarfs}

In addition to allocating fibres to supernova hosts, we also allocated fibres to candidate white dwarfs and to potential 

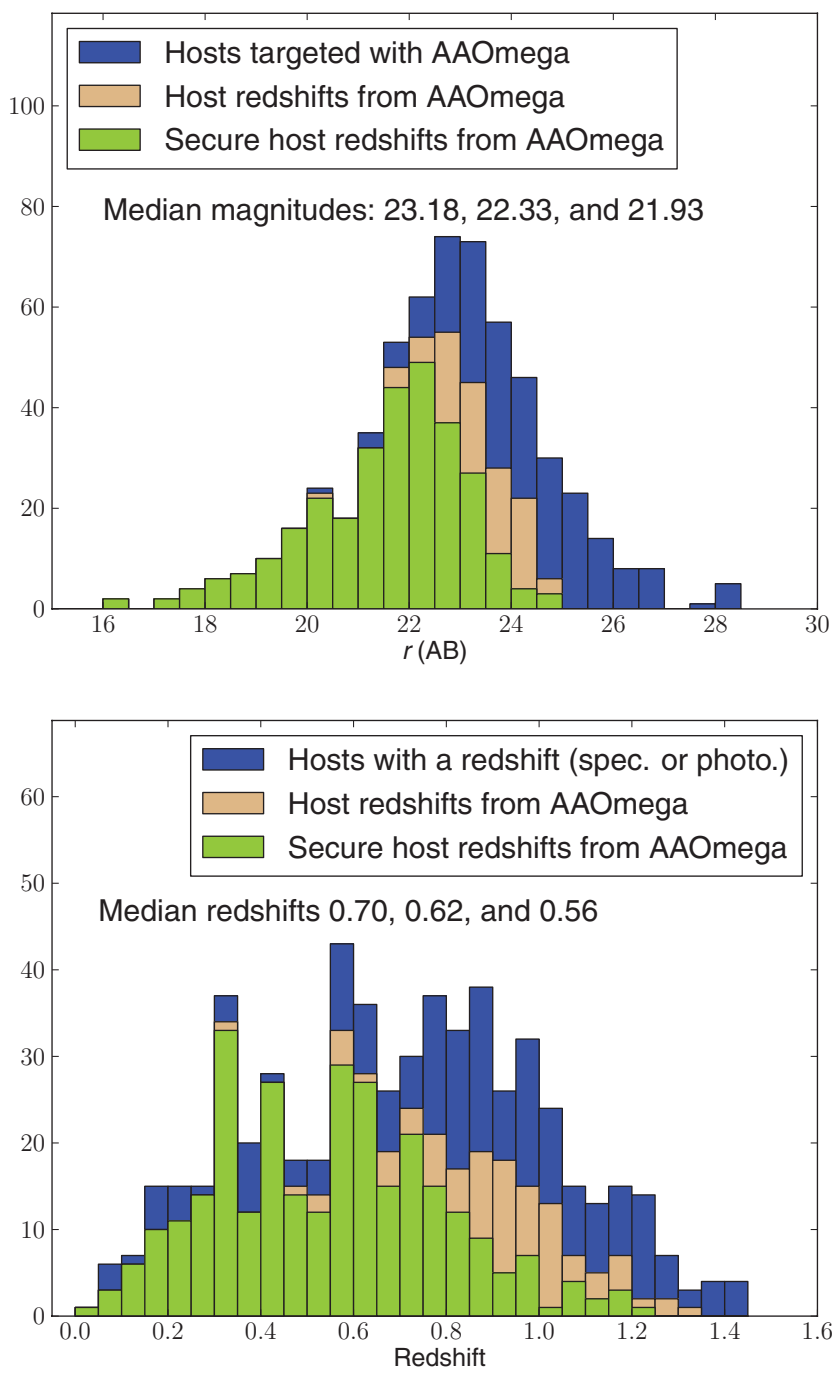

Figure 1. Histograms of the number of objects plotted as a function of magnitude (top) and redshift (bottom). The blue, green, and tan histograms represent all objects targeted with AAOmega, all objects with secure AAOmega redshifts (quality flags 4 or 5), and all objects with either a secure AAOmega redshift or a probable one (quality flags 3,4 , or 5). If the $r$-band magnitude of the host was unavailable, then the object was not plotted in the upper plot. If a spectroscopic redshift is not available for the lower plot, we use the photometric one. If neither a spectroscopic nor a photometric redshift were available, then the object was not plotted in the lower panel. The median magnitudes and redshifts of objects in the blue, tan, and green histograms annotate each figure.

cluster galaxies. We defer the discussion on the follow-up of cluster galaxies until the next section: here we discuss the selection of white dwarfs.

White dwarfs can be easily identified in the SNLS data, using either their location in colour-colour plots or their relatively high proper motions. Both the D1 and D4 fields were searched for white dwarfs using both techniques. A listing of the candidates, notes on how they were found, and the spectral classification that we have assigned to each of the objects are listed in Table 3.
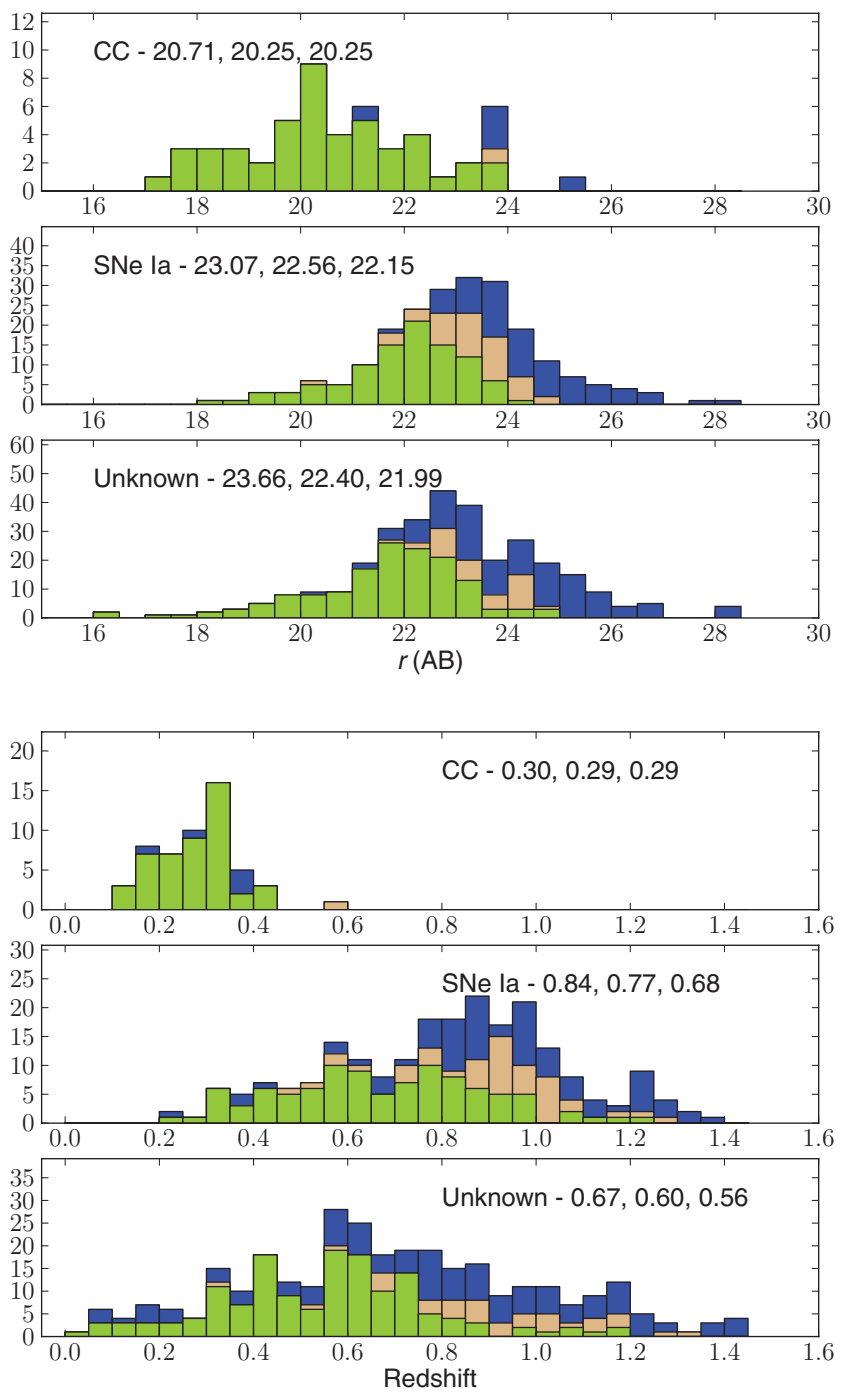

Figure 2. As for Figure 1, but split according to the photometric classification.

Limboz et al. (2008) also searched the SNLS fields for white dwarfs. The criteria they use to select white dwarfs differ from ours, so their catalogue and ours are not identical. They use different cuts in colour-colour space, different magnitude limits, and did not use proper motions. However, nearly all of our candidates were also found by them. The candidates in common are marked with the letter ' $a$ ' in Table 3. Since none of the Limboz et al. (2008) candidates in the D1 and D4 fields had been spectroscopically confirmed, we assigned fibres to all but one of objects that were in their catalogue but not in ours. These objects are marked in Table 3 with the letter ' $b$ '. Not all white dwarf candidates were targeted for spectroscopy. We avoided the brightest one from Limboz et al. (2008) because of concerns that crosstalk between fibres would contaminate neighbouring spectra.

Out of 14 candidates, 12 are white dwarfs and 2 are AGNs. Of the 12 white dwarfs, we classify nine as DA white dwarfs, two as DC white dwarfs, and one as a possible magnetic white 
Table 3. Candidate White Dwarfs in the SNLS D1 and D4 Fields

\begin{tabular}{lccll}
\hline \hline $\begin{array}{l}\text { RA } \\
\text { (J2000) }\end{array}$ & Dec. & $\begin{array}{c}g \text { Band Mag } \\
(\mathrm{AB})\end{array}$ & \multicolumn{1}{c}{ Discovery Method } & Classification \\
\hline 022511.64 & $-045610.6^{a}$ & 17.77 & Colours and proper motion & DA \\
022405.27 & $-042216.3^{a}$ & 17.90 & Proper motion only & DA \\
022746.39 & $-042109.2^{a}$ & 19.86 & Colours and proper motion & DC \\
022558.19 & $-040236.5^{a}$ & 19.56 & Colours and proper motion & DA \\
022423.76 & $-041318.7^{b}$ & 19.33 & Colours only & DA \\
022512.69 & $-043851.3^{b}$ & 21.14 & Colours only & DA \\
022734.20 & $-042228.6^{b}$ & 20.41 & Colours only & AGN $z=1.14$ \\
221458.39 & $-180742.6^{a}$ & 20.39 & Colours and proper motion & DA \\
221405.75 & $-175905.2^{a}$ & 19.27 & Colours and proper motion & DAH? \\
221621.93 & $-174407.9^{a}$ & 20.31 & Colours only & DA \\
221410.93 & $-174036.5^{a}$ & 19.91 & Colours only & DA \\
221326.42 & $-172318.9^{a}$ & 20.37 & Colours and proper motion & DA \\
221400.06 & -172146.1 & 19.77 & Colours only & AGN $z=2.10$ \\
221330.98 & $-180144.5^{b}$ & 20.99 & Colours only & DC \\
\hline \hline
\end{tabular}

${ }^{a}$ Also listed in Limboz et al. (2008).

${ }^{b}$ From Limboz et al. (2008).

Table 4. Cluster Redshifts

\begin{tabular}{lccc}
\hline \hline Cluster & $\begin{array}{c}\text { Cluster } \\
\text { Class }^{a}\end{array}$ & $\begin{array}{c}\text { Mean } \\
\text { Redshift }\end{array}$ & $\begin{array}{c}\text { Number of } \\
\text { Redshifts }\end{array}$ \\
\hline XLSS J022357.4-043516 & C1 & 0.495 & 4 \\
XLSS J022404.1-041330 & C1 & 1.051 & 1 \\
XLSS J022303.3-043621 & C2 & $\cdots$ & 0 \\
\hline \hline
\end{tabular}

${ }^{a}$ See Pierre et al. (2007).

dwarf (DAH). The two AGNs were both selected from their colours and were not in the sample that used proper motions in the selection criteria. At high galactic latitudes, AGNs start to outnumber white dwarfs by $g=17$ (Fan 1999), so they are an important source of contamination.

In Section 6.3, we discuss how DA white dwarfs might be used as an alternative means of calibrating SN fluxes.

\subsection{Cluster Galaxies}

For the D1 field, we assembled a catalogue of 13 galaxies in three X-ray-selected clusters from the XMM-LSS survey (Adami et al. 2011), which covers the entire D1 field. Out of 13 galaxies, seven were assigned fibres and six redshifts were obtained. The results are summarised in Table 4 . Both XLSS J022404.1-041330 and XLSS J022303.3-043621at $z=1.05$ and 1.22 , respectively-had been confirmed with data from other facilities (Adami et al. 2011). Our observations reconfirm the redshift of XLSS J022404.1-041330 but failed to do so for XLSS J022303.3-043621. Prior to our observations, only one galaxy redshift had been measured for XLSS J022357.4-043516 (Adami et al. 2011). Our observations add three more redshifts to this cluster.

\section{DISCUSSION}

\subsection{A Comparison with the Real-Time Follow-up}

It is instructive to compare the results we obtain here with the results that we obtained with the Very Large Telescope, VLT during the real-time follow-up of live candidates. During the final 2 years of the spectroscopic follow-up of candidates with the VLT, the Multi-Object Spectroscopy modes of FORS1 and FORS2 were used to observe both live candidates and the host galaxies of other transients that were discovered in earlier years. By the end of the SNLS survey, there were typically three to four host galaxies visible in the FORS $7 \times$ $7 \operatorname{arcmin}^{2}$ FoV.

The MOS modes of FORS1 and FORS2 consists of 38 movable blades that can be used to make 19 slits anywhere in the FORS focal plane. While there is a mode that allows pre-cut masks to be inserted into the FORS2 focal plane (called the MXU mode), this mode was not available for target-of-opportunity requests.

A number of targets were observed with both the VLT and AAOmega. Out of the 83 targets that were observed with both instruments, 63 have a redshift from both AAOmega and the VLT. The redshifts of all objects agree to within 0.002. The uncertainties in the redshifts from FORS1 and FORS2 are around 0.001 and are generally larger than the uncertainties in the redshifts from AAOmega.

In Figure 3, we compare the magnitude and redshift distributions of objects that were observed with FORS1 and FORS2 with those that were observed with AAOmega. Excluding any sort of renormalisation to account for differences in exposure times, observing efficiency, and target selection, the redshift distributions are broadly similar.

The redshifts from FORS1 and FORS2 came from 66 separate MOS set-ups. The exposure time for a single set-up was typically $1 \mathrm{~h}$. Summed over all set-ups, the total amount 

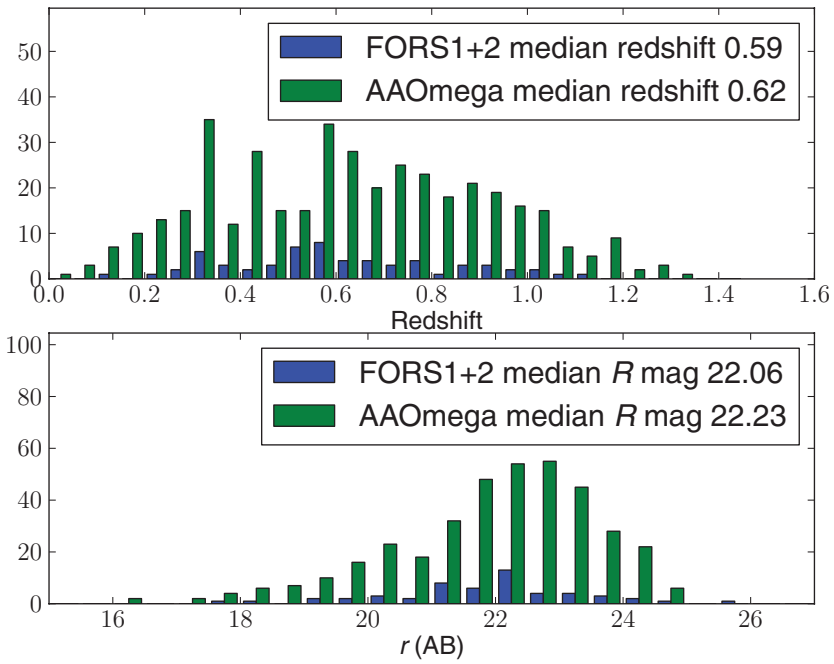

Figure 3. A comparison of the host redshift and magnitude distributions obtained with FORS1 and FORS2 with the host and magnitude distributions obtained with AAOmega.

of time spent on the D1 and D4 fields with FORS1 and FORS2 was $240000 \mathrm{~s}$. The integration times that were used with AAOmega for the D1 and D4 fields were 32500 and $60000 \mathrm{~s}$, respectively.

At face value, it would seem that AAOmega - a multiobject spectrograph on a 4-m class telescope-has resulted in many more redshifts than FORS1 and FORS2-both multiobject spectrographs on $8-\mathrm{m}$ class telescopes. While this is true, the difference is not as extreme as that suggested by inspecting Figure 3, since the number of targets available for the AAOmega follow-up was about a factor of 2 larger than the number available for FORS1 and FORS2. Nevertheless, it is difficult to imagine that the efficiency with FORS could have been increased by more than a factor of 3 if we were to repeat the experiment with FORS2 with the MXU mode and with the catalogue that was used in the follow-up with AAOmega. The main reason for the difference comes from the difference in the FoV between AAOmega and FORS2. With AAOmega, we can cover the entire 1-deg ${ }^{2}$ MegaCam field in one shot, which is 60 times the area covered by FORS2. This more than compensates for differences in telescope aperture and image quality.

While AAOmega is efficient in obtaining large numbers of host galaxy redshifts, it is not suitable for confirming SN types-which was, together with obtaining redshifts, the main aim of the observations with the VLT - over the redshift range that was probed with FORS1 and FORS2.

\subsection{Redshifts: Photometry versus Spectroscopy}

Photometric redshifts are often used as an alternative to spectroscopic redshifts when samples become large or when the precision and accuracy provided by photometric redshifts are sufficient for the tasks at hand. They also allow redshifts to be obtained for sources that are too faint for spectroscopy. For

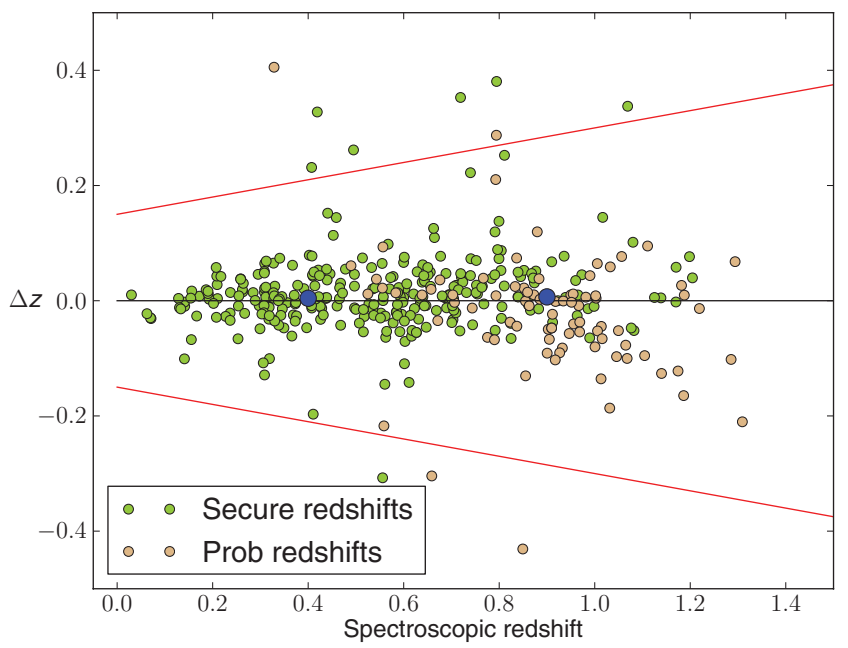

Figure 4. The deviation $\Delta z$ between spectroscopic and photometric measures of redshift plotted against the spectroscopic redshift for objects with secure and probable spectroscopic redshifts (the green and tan circles, respectively). The median of the difference-in bins of width 0.5 in redshift, centred at $z=0.4$ and 0.9 - are plotted as the large blue circles. The horizontal black line represents equality between the photometric and spectroscopic redshifts. The red lines represent the boundary beyond which the photometric redshift differs from the spectroscopic one by more than $0.15(1+z)$.

some future transient surveys, such as the transient survey of the Large Synoptic Survey Telescope (LSST), photometrically measured redshifts of the hosts of the transients will be the only realistic option for obtaining redshifts for most of the transients.

Photometric redshifts are both less precise and less accurate (in the sense that they are biased) than spectroscopic redshifts. Up to $z=1$, the precision of photometric redshifts, as measured by the normalised median absolute deviation$\langle|\Delta z| /(1+z)\rangle$, (Ilbert et al. 2006) - is typically around 0.03 . This can be compared to the level of uncertainty that is typical for redshifts that are measured spectroscopically, which is generally better than 0.001 .

The lower precision appears amenable to solution by simply increasing the number of SNe Ia in the sample. The issue of accuracy is more critical, since any change in the accuracy with redshift might be interpreted as evolution in the dark energy equation-of-state parameter.

In Figure 4, we compare the photometric redshifts (from Ilbert et al. 2006) assigned to our targets with the spectroscopic redshifts measured in this paper with AAOmega. Objects with secure and probable redshifts are represented by the green and tan symbols, respectively.

To estimate the accuracy of the photometric redshifts, we compute the median difference between spectroscopic and photometric measures of the redshift in two broad redshift bins centred at $z=0.4$ and 0.9 and plot them as blue circles in Figure 4. The values in these two bins are 0.004 and 0.007 , respectively, with uncertainties, which are estimated via bootstrap resampling, of 0.002 for both bins. In the standard $\Lambda \mathrm{CDM}$ (Cold Dark Matter) cosmology, an offset of 0.007 in redshift at $z=0.9$ corresponds to a change of $1 \%$ 
in luminosity, which changes the best-fit value for the dark energy equation-of-state parameter by about $4 \%$. In SN Ia samples that are currently used for cosmology, the systematic error in the dark energy equation-of-state parameter is around 6\% (Conley et al. 2011; Sullivan et al. 2011). Samples that use photometric redshifts for host galaxies instead of spectroscopic redshifts will need to manage a source of systematic error that is roughly similar in magnitude to the systematic error in current samples.

In principle, one could remove the offset in the photometric redshifts using the spectroscopic redshifts to work out the size of the offset, as we have done here. With about 160 redshifts (the number of redshifts that go into computing the location of the blue point at $z=0.9$ in Figure 4), the uncertainty in the correction is 0.002 , which at $z=0.9$ corresponds to a $1 \%$ error in the dark energy equation-of-state parameter. With significantly more redshifts, the uncertainty could be made smaller still.

An interesting alternative to using redshifts of the host galaxies to determine the size of the offset, which we do here, is to use redshifts of general field galaxies, since these are more numerous. However, there may be serious limitations to this approach. At some level, the offset must depend on galaxy type. For example, the offset for galaxies with strong emission lines will be different for galaxies that lack emission lines. So estimates of the size of the offset may be erroneous if the population of galaxies that hosted SNe Ia differs from population of galaxies that is used to determine the offset.

Photometric redshifts are also prone to catastrophic failures, which lead to non-Gaussian tails in the normalised redshift deviation: $\Delta z /(1+z)$. A commonly used definition for catastrophic failures is when the photometric redshift differs from the spectroscopic one by more than $0.15(1+z)$ (Ilbert et al. 2006). We can use our redshifts from AAOmega to make an independent measurement of the rate of catastrophic failures. Considering secure and probable redshifts together, the rate is $2.9 \%$, which is similar to the $3.7 \%$ rate reported in Ilbert et al. (2006).

\subsection{DA White Dwarfs as In-Situ Flux Calibrators}

Systematic errors now contribute as much to the uncertainty in the dark energy equation-of-state parameter as statistical errors (Conley et al. 2011; Sullivan et al. 2011; Suzuki et al. 2011). The largest systematic error, by far, comes from calibration (Conley et al. 2011; Sullivan et al. 2011), which in simple terms is the process of converting the observed counts in some filter to a relative flux. For the purpose of constraining cosmology, it is not necessary to know the absolute flux.

DA (pure hydrogen) white dwarfs are commonly used as flux calibrators. For example, the primary spectrophotometric standards of the Hubble Space Telescope (HST) are three DA white dwarfs (Bohlin 2000). The absolute precision of the spectral energy distributions (SEDs) of these stars is around $1 \%$ in the optical and near-infrared (Bohlin 2007). The precision of DA white dwarf models, which use spectral measurements of the effective gravity and temperature, is thought to be even greater (Allende Prieto, Hubeny, \& Smith 2009).

The idea of extending the HST network of primary standards to more DA white dwarfs is explored in Allende Prieto et al. (2009). From an initial sample of 598 objects from the Sloan Digital Sky Survey (SDSS), they build a sample of 57 suitable DA white dwarfs (where suitable means a sufficiently small $\chi^{2}$ in the model fit to the SED, and model parameters $21000<T_{\text {eff }}<85000$ and $7.1<\log g<9$ ) that are then analysed in more detail. This sample is then reduced to nine after taking into account all sources of error, including extinction, which becomes important for more distant objects.

In the two 1-deg ${ }^{2}$ SNLS fields that we observed, we found four DA white dwarfs per field down to $g_{\mathrm{AB}} \sim 20$ (see Section 5.2). For DES (Bernstein et al. 2011), which has a FoV that is three times larger, there will be $\sim 12$ DA white dwarfs to this magnitude limit. For SkyMapper (Keller et al. 2007), which has an even larger FoV, there will be around 25 DA white dwarfs in regions that have a similar galactic latitude to the DES and SNLS fields, and considerably more at low galactic latitudes.

While it is encouraging that there are a sufficient number of DA white dwarfs for one to consider the possibility of using DA white dwarfs as in-situ calibrators, the key issue is whether or not the space density of suitable white dwarfs is sufficient. Scaling from the numbers reported in Allende Prieto et al. (2009) would suggest that the answer to this is question is no. However, the needs for supernova cosmology may be less stringent, since only the relative calibration of the SED as a function of wavelength and the relative normalisation of the SEDs matters. Furthermore, one can average the results over the white dwarfs that are within the field.

\subsection{Selection Biases}

The SNLS 3-year constraints on the dark energy equation-ofstate parameter used SNe Ia that were discovered in real time and subsequently confirmed to be SNe Ia with spectroscopy of the supernovae near maximum light. For simplicity, we will refer to these SNe Ia as the SNLS 3-year sample. The realtime discovery of candidates and the assignment of priorities for spectroscopic follow-up are described in Howell et al. (2005), Sullivan et al. (2006a), and Perrett et al. (2010).

The criteria used to select and classify candidates in Bazin et al. (2011) differ from those used for the SNLS 3year sample. Furthermore, the spectroscopy undertaken with AAOmega is not used to confirm the supernova type, as was done for SNe Ia in the SNLS 3-year sample, but to obtain the host redshift. Hence, biases incurred in using a SN Ia sample that uses photometrically classified SNe Ia with host galaxy redshifts (we will refer to this sample as the host-z sample) to constrain the dark energy equation-of-state will differ from the biases that affect the SNLS 3-year sample. 
Perrett et al. (2010) make a detailed analysis of the biases incurred in the SNLS 3-year sample due to incomplete coverage of the underlying $\mathrm{SNe}$ Ia population. In this section, our aim is not to extend this work to the host- $z$ sample but to describe how the biases are likely to differ between the two samples. Nevertheless, such a study will be necessary if the host- $z$ sample is to be used to constrain cosmological parameters.

Selection biases are incurred at three stages: the initial photometric selection of candidates, the spectroscopic follow-up, and the application of selection cuts to the fully assembled and integrated data set. We briefly discuss the biases associated with the photometric selection of candidates first, then discuss the biases associated with the spectroscopic followup in more detail, and end with a comment on the impact of selection cuts.

While the photometric selection of SNe Ia in the two samples differs, the nature of the selection bias is the same for both samples. Supernovae are not perfect standard candles; hence, magnitude-limited samples (as both these samples are) will tend to select the brightest events as the magnitude limit of the survey is approached (Malmquist bias). Once assumptions are made about the properties of the parent $\mathrm{SNe}$ Ia population and how these properties evolve with redshift, the bias can be computed with Monte Carlo simulations (Perrett et al. 2010). At a given redshift, the photometric sample of Bazin et al. (2011) is less biased than the SNLS 3-year sample, because it has a fainter magnitude limit.

The biggest difference between the two samples lies with the spectroscopic follow-up. For the SNLS 3-year sample, the spectroscopic follow-up can be broken into three steps: (i) the real-time sample is filtered for $\mathrm{SNe}$ Ia that are deemed to be suitable for spectroscopy; (ii) a request for spectroscopy is made and a spectrum is taken; and (iii) the supernova is identified.

The bias incurred from the last step is probably the most difficult to model. One well-known difficulty in positively identifying SNe Ia spectroscopically is that SNe Ia in bright hosts are more difficult to classify than SNe Ia in faint hosts. The difficulty increases with redshift. The effect is similar to that imposed by the percentage increase cut, which is used in the SNLS 3-year sample. Imposing this cut at the stage of selecting which candidates go on to be observed spectroscopically makes computing the bias more tractable, as part of the bias can be modelled using the imaging data.

The bias that results from the spectroscopic follow-up of host galaxies in the host- $z$ sample is different. It is generally much easier to obtain the redshift of a galaxy if it is bright, if it is forming stars, or if it recently did so (such poststarburst galaxies may lack strong emission lines but contain clear Balmer absorption lines due to the presence of large numbers of A-type stars). If the properties of SNe Ia were independent of the properties of their hosts, then it would not matter if it was easier to get redshifts for these galaxies. However, there is ample evidence that the properties of SNe Ia do depend on the properties of their hosts. Brighter SNe
Ia with broader light curves are preferentially found in starforming galaxies (Hamuy et al. 1995, 2000; Sullivan et al. 2006b), and brighter SNe Ia also tend to live in more massive (and therefore brighter) galaxies (see Sullivan et al. 2010, for example).

This selection bias might be large enough to substantially affect the accuracy of future experiments, such as the DES SN survey. However, with a sufficiently large enough sample of host galaxies, it should be possible to model the probability of getting a host redshift given the photometric properties of the host.

There is also the possibility that the wrong host galaxy is identified. Fortunately, this seems to be relatively rare. Dawson et al. (2009) show an example where the redshift of what appears to be the likely host disagrees with that of the supernova. Another interesting example is SNLS-P04D1ay from this paper. SNLS-P04D1ay was observed with FORS2 and AAOmega. Two sets of lines at different redshifts $(z=0.446$ and 0.905 ) could be identified in the spectra from both instruments. This is a clear case of two galaxies along the same line of sight. A potential way to eliminate some of these events would be to exclude events in which the photometric redshift from either the supernova or the host disagrees with the spectroscopic one by an amount that does not exclude too many supernovae that have been correctly associated with their hosts.

Finally, before the fully assembled $\mathrm{SNe}$ Ia data set is used for cosmology-or for other studies, such as supernova rates-selection cuts for quality are applied. Usually, this means that for every supernova there needs to be a certain number of observations with a given signal-to-noise ratio in a certain number of filters both before and after maximum light. For the SNLS 3-year sample, the selection cuts reduce the number of SNe used for cosmology from 279 to 242 (Conley et al. 2011). Similar selection cuts are applied to the host- $z$ sample; however, these cuts usually occur during the initial selection.

\section{SUMMARY AND CONCLUSIONS}

Using the $2 \mathrm{dF}$ fibre positioner and the AAOmega spectrograph on the AAT, we observed 698 galaxies in two SNLS fields that hosted supernovae during the first 3 years of the SNLS. With integrations varying from 10 to $60 \mathrm{ks}$ per object, we obtained redshifts for 400 of them. The redshifts cover a very broad range, from $z=0.03$ to 1.3 .

The percentage of fibres allocated to host galaxies was very high. Even though the number of supernova hosts outnumbered the number of fibres by as little as $10 \%$, we were able to allocate almost $100 \%$ of the fibres that were free to observe supernova hosts. This was achieved with the additional restriction that all the hosts were located in the central $1-\mathrm{deg}^{2}$ of the $2 \mathrm{dF}$ FoV.

The median redshift of photometrically classified SNe Ia in our sample is $z \sim 0.77$, which is higher than the median redshift of SNe Ia in the SNLS 3-year SNe Ia sample 
$(z=0.61)$ and higher than the expected median redshift of SNe Ia in the upcoming DES supernova survey.

The DES supernova search will discover around 4000 $\mathrm{SNe}$ Ia in the redshift range of $0.2<z<1.2$ over a period of approximately 5 years (Bernstein et al. 2011). The number of discoveries is an order of magnitude larger than the number of SNe Ia found in either the SDSS supernova survey or the SNLS. Spread over 10 fields, this amounts to 400 SNe Ia per field. After 5 years of the operation, the number of SNe Ia per DES supernova field will be similar to the number of fibres in $2 \mathrm{dF}$.

Obtaining the redshifts of galaxies that hosted photometrically classified $\mathrm{SNe}$ with a multi-object fibre-fed facility, such AAOmega on the AAT, is an efficient alternative to obtaining a spectrum of every supernova, one at a time, while it is bright enough to do so. However, before photometrically classified SN Ia samples can be used to constrain cosmology, the biases in them need to be examined with the level of rigour that is currently done for spectroscopically confirmed samples.

\section{ACKNOWLEDGMENTS}

The authors thank the XMM-LSS collaboration, in particular Nicolas Cleric and Marguerite Pierre, for providing the coordinates of galaxies in three X-ray-selected galaxy clusters in the D1 field. Chris
Lidman acknowledges the support from the Australian Research Council (ARC) Future Fellowship Grant (FT0992259) and the excellent support provided by the staff at the Australian Astronomical Observatory. The Centre for All-sky Astrophysics (CAASTRO) is an Australian Research Council Centre of Excellence, funded by grant CE11E0090. Mark Sullivan acknowledges the support of the Royal Society.

\section{APPENDIX: REDSHIFT CATALOGUE}

The redshift catalogue is presented in Table 5. Only galaxies that have a redshift quality flag of 3 or higher are listed. The supernovae that correspond to these galaxies are given names that follow the convention SNLS-PyyDnxx. SNLS-P is the prefix indicating that the supernova was found by Bazin et al. (2011), yy marks the year during which it occurred, Dn marks the SNLS field in which it occurred (e.g. D1 or D4), and $x x$ starts off as $a a$ for the first supernova in a given year and field and increments as $a a, a b$, $\ldots, a z, b a, \ldots$, etc. for new supernova. If the supernova matches an object in the real-time database (RTD), the RTD name is also given. Redshift uncertainties are generally less than 0.001 . The coordinates refer to the location where the fibre was placed. In most cases, this corresponds to the supernova host. In cases where there was no clear host, the fibre was placed at the location of the supernova.

Table 5. AAOmega Host Galaxy Redshifts of Photometrically Identified Events

\begin{tabular}{|c|c|c|c|c|c|}
\hline Name & RTD Name & RA (J2000) & Dec. (J2000) & Redshift & Quality Flag \\
\hline SNLS-P03D1ad & $\ldots$ & 022607.46 & -044016.3 & 0.724 & 4 \\
\hline SNLS-P03D1af & $\ldots$ & 022428.52 & -040838.2 & 1.083 & 4 \\
\hline SNLS-P03D1ah & SNLS-03D1be & 022551.20 & -041602.8 & 0.825 & 4 \\
\hline SNLS-P03D1ah & SNLS-03D1br & 022609.42 & -042345.9 & 0.426 & 5 \\
\hline SNLS-P03D1aj & SNLS-03D1am & 022413.84 & -042602.3 & 0.557 & 3 \\
\hline SNLS-P03D1ak & SNLS-03D1aq & 022503.10 & -040501.9 & 0.705 & 5 \\
\hline SNLS-P03D1al & SNLS-03D1bk & 022627.49 & -043211.7 & 0.866 & 4 \\
\hline SNLS-P03D1an & SNLS-03D1ax & 022423.24 & -044316.0 & 0.497 & 4 \\
\hline SNLS-P03D1ao & $\ldots$ & 022540.15 & -041847.0 & 0.596 & 5 \\
\hline SNLS-P03D1ap & $\ldots$ & 022648.20 & -040353.4 & 0.548 & 4 \\
\hline SNLS-P03D1ar & $\ldots$ & 022453.34 & -041616.8 & 0.648 & 4 \\
\hline SNLS-P03D1ay & SNLS-03D1by & 022754.02 & -040304.4 & 0.378 & 5 \\
\hline SNLS-P03D1bg & $\ldots$ & 022447.80 & -043102.0 & 0.584 & 3 \\
\hline SNLS-P03D1bh & SNLS-03D1df & 022641.90 & -044513.1 & 0.237 & 4 \\
\hline SNLS-P03D1bi & $\ldots$ & 022621.97 & -045030.2 & 0.704 & 4 \\
\hline SNLS-P03D1br & $\ldots$ & 022442.89 & -042103.2 & 1.294 & 3 \\
\hline SNLS-P03D1bu & $\ldots$ & 022424.26 & -044426.4 & 0.142 & 5 \\
\hline SNLS-P03D1by & SNLS-03D1du & 022459.84 & -041838.2 & 0.852 & 3 \\
\hline SNLS-P03D1ca & $\ldots$ & 022651.58 & -041415.0 & 0.210 & 4 \\
\hline SNLS-P03D1cb & $\ldots$ & 022521.28 & -042300.0 & 0.602 & 4 \\
\hline SNLS-P03D1cd & SNLS-03D1fb & 022712.84 & -040716.7 & 0.497 & 4 \\
\hline SNLS-P03D1cg & $\ldots$ & 022748.49 & -041901.6 & 0.689 & 4 \\
\hline SNLS-P03D1ck & SNLS-03D1ea & 022750.35 & -040502.2 & 0.313 & 5 \\
\hline SNLS-P03D1cp & $\ldots$ & 022706.13 & -043128.3 & 0.986 & 4 \\
\hline SNLS-P03D1ct & $\ldots$ & 022429.11 & -040955.0 & 0.265 & 5 \\
\hline SNLS-P03D1cu & $\ldots$ & 022432.61 & -043003.2 & 0.328 & 5 \\
\hline SNLS-P03D1cv & $\ldots$ & 022508.57 & -043825.1 & 0.366 & 5 \\
\hline SNLS-P03D1cw & SNLS-03D1gi & 022518.12 & -043155.7 & 0.525 & 3 \\
\hline SNLS-P03D4aa & $\ldots$ & 221637.85 & -175520.5 & 0.562 & 5 \\
\hline SNLS-P03D4aa & SNLS-03D4at & 221424.01 & -174636.0 & 0.634 & 4 \\
\hline
\end{tabular}


Table 5. Continued.

\begin{tabular}{|c|c|c|c|c|c|}
\hline Name & RTD Name & RA (J2000) & Dec. (J2000) & Redshift & Quality Flag \\
\hline SNLS-P03D4ab & SNLS-03D4az & 221547.79 & -180751.2 & 0.409 & 5 \\
\hline SNLS-P03D4ab & SNLS-03D4ev & 221651.39 & -172003.1 & 0.538 & 4 \\
\hline SNLS-P03D4ac & $\ldots$ & 221354.79 & -171711.4 & 0.408 & 5 \\
\hline SNLS-P03D4ac & $\ldots$ & 221654.51 & -180212.1 & 0.936 & 4 \\
\hline SNLS-P03D4ad & SNLS-03D4da & 221557.04 & -180521.4 & 0.328 & 5 \\
\hline SNLS-P03D4ae & SNLS-03D4au & 221609.92 & -180439.2 & 0.469 & 5 \\
\hline SNLS-P03D4af & SNLS-03D4fd & 221614.44 & -172343.4 & 0.791 & 3 \\
\hline SNLS-P03D4ag & $\ldots$ & 221549.67 & -174331.7 & 0.755 & 4 \\
\hline SNLS-P03D4ai & $\ldots$ & 221605.63 & -172623.3 & 1.077 & 4 \\
\hline SNLS-P03D4aj & SNLS-03D4aa & 221656.37 & -175738.1 & 0.167 & 5 \\
\hline SNLS-P03D4ak & SNLS-03D4fy & 221533.16 & -174414.3 & 0.339 & 5 \\
\hline SNLS-P03D4al & & 221526.85 & -171600.3 & 0.643 & 5 \\
\hline SNLS-P03D4am & SNLS-03D4gg & 221640.22 & -180951.0 & 0.593 & 4 \\
\hline SNLS-P03D4an & SNLS-03D4bc & 221528.21 & -174948.1 & 0.573 & 4 \\
\hline SNLS-P03D4ao & SNLS-03D4ag & 221445.85 & -174421.9 & 0.285 & 5 \\
\hline SNLS-P03D4ao & SNLS-03D4gh & 221534.59 & -175610.4 & 0.341 & 5 \\
\hline SNLS-P03D4ap & SNLS-03D4hd & 221539.43 & -175337.4 & 0.872 & 4 \\
\hline SNLS-P03D4aq & SNLS-03D4ai & 221641.54 & -172610.4 & 0.202 & 5 \\
\hline SNLS-P03D4ar & SNLS-03D4be & 221409.07 & -173638.9 & 0.414 & 5 \\
\hline SNLS-P03D4as & $\ldots$ & 221441.49 & -173213.7 & 0.338 & 4 \\
\hline SNLS-P03D4au & $\ldots$ & 221543.69 & -174835.6 & 0.800 & 4 \\
\hline SNLS-P03D4av & $\ldots$ & 221458.89 & -175044.1 & 0.313 & 5 \\
\hline SNLS-P03D4aw & SNLS-03D4bj & 221430.09 & -180649.9 & 0.895 & 3 \\
\hline SNLS-P03D4ay & & 221455.22 & -172430.3 & 0.602 & 5 \\
\hline SNLS-P03D4az & SNLS-03D4bx & 221448.64 & -173117.7 & 0.600 & 4 \\
\hline SNLS-P03D4ba & SNLS-03D4bf & 221701.34 & -180042.9 & 0.805 & 5 \\
\hline SNLS-P03D4bb & SNLS-03D4cd & 221718.42 & -180126.4 & 0.845 & 4 \\
\hline SNLS-P03D4bc & SNLS-03D4bl & 221405.44 & -171838.1 & 0.319 & 5 \\
\hline SNLS-P03D4bf & SNLS-03D4cw & 221611.46 & -171359.7 & 0.155 & 5 \\
\hline SNLS-P03D4bh & SNLS-03D4cn & 221634.56 & -171613.6 & 0.818 & 3 \\
\hline SNLS-P03D4bi & $\ldots$ & 221638.23 & -173650.8 & 1.006 & 3 \\
\hline SNLS-P03D4bl & $\ldots$ & 221710.80 & -172408.0 & 1.205 & 4 \\
\hline SNLS-P03D4bt & SNLS-03D4cy & 221340.47 & -174055.1 & 0.927 & 3 \\
\hline SNLS-P03D4bu & SNLS-03D4cz & 221641.80 & -175534.5 & 0.697 & 4 \\
\hline SNLS-P03D4bw & SNLS-03D4ed & 221619.76 & -173127.5 & 0.860 & 3 \\
\hline SNLS-P03D4by & SNLS-03D4di & 221410.24 & -173024.3 & 0.905 & 3 \\
\hline SNLS-P03D4bz & SNLS-03D4dh & 221731.08 & -173747.6 & 0.627 & 5 \\
\hline SNLS-P03D4cb & $\ldots$ & 221331.21 & -173014.2 & 1.310 & 3 \\
\hline SNLS-P03D4cd & SNLS-03D4ec & 221443.70 & -172140.6 & 1.016 & 3 \\
\hline SNLS-P03D4cf & $\ldots$ & 221327.50 & -174005.0 & 0.368 & 4 \\
\hline SNLS-P03D4ch & SNLS-03D4dp & 221539.04 & -171652.4 & 0.955 & 3 \\
\hline SNLS-P03D4cj & $\ldots$ & 221516.12 & -172048.2 & 0.540 & 5 \\
\hline SNLS-P03D4ck & SNLS-03D4gi & 221708.68 & -18 1246.4 & 0.578 & 4 \\
\hline SNLS-P03D4cl & $\ldots$ & 221658.24 & -171832.8 & 1.068 & 3 \\
\hline SNLS-P04D1aa & SNLS-04D1ad & 022415.96 & -042844.9 & 0.392 & 5 \\
\hline SNLS-P04D1ab & $\ldots$ & 022708.48 & -041116.9 & 0.157 & 4 \\
\hline SNLS-P04D1ac & $\ldots$ & 022509.52 & -041041.5 & 1.003 & 3 \\
\hline SNLS-P04D1ac & SNLS-04D1ae & 022557.33 & -043321.8 & 0.617 & 4 \\
\hline SNLS-P04D1ag & SNLS-04D1ec & 022415.25 & -042308.6 & 0.603 & 4 \\
\hline SNLS-P04D1ai & SNLS-03D1gr & 022559.00 & -040928.2 & 0.296 & 5 \\
\hline SNLS-P04D1aj & $\ldots$ & 022701.41 & -041530.7 & 0.528 & 5 \\
\hline SNLS-P04D1al & $\ldots$ & 022723.85 & -040437.5 & 0.623 & 5 \\
\hline SNLS-P04D1am & $\ldots$ & 022537.74 & -043549.1 & 0.612 & 4 \\
\hline SNLS-P04D1am & $\ldots$ & 022608.55 & -043133.5 & 0.823 & 3 \\
\hline SNLS-P04D1an & SNLS-04D1fh & 022659.38 & -042942.3 & 0.687 & 4 \\
\hline SNLS-P04D1ap & SNLS-04D1de & 022635.93 & -042521.8 & 0.768 & 4 \\
\hline SNLS-P04D1au & SNLS-04D1hw & 022513.39 & -045202.0 & 0.350 & 4 \\
\hline SNLS-P04D1aw & $\ldots$ & 022739.16 & -041102.1 & 0.718 & 4 \\
\hline SNLS-P04D1ax & SNLS-04D1hb & 022620.26 & -040745.5 & 0.912 & 3 \\
\hline SNLS-P04D1ay & SNLS-04D1dz & 022753.68 & -040512.1 & 0.446 & 5 \\
\hline SNLS-P04D1be & $\ldots$ & 022536.24 & -043616.1 & 0.825 & 4 \\
\hline SNLS-P04D1bg & SNLS-04D1la & 022639.80 & -044953.0 & 0.319 & 4 \\
\hline SNLS-P04D1bi & SNLS-04D1hk & 022445.94 & -042405.1 & 0.635 & 4 \\
\hline
\end{tabular}


Table 5. Continued.

\begin{tabular}{|c|c|c|c|c|c|}
\hline Name & RTD Name & RA (J2000) & Dec. (J2000) & Redshift & Quality Flag \\
\hline SNLS-P04D1bj & SNLS-04D1iu & 022431.30 & -040329.6 & 0.254 & 5 \\
\hline SNLS-P04D1bk & SNLS-04D1jg & 022612.64 & -040807.1 & 0.585 & 4 \\
\hline SNLS-P04D1bl & SNLS-04D1jt & 022547.62 & -042232.8 & 0.639 & 5 \\
\hline SNLS-P04D1bn & SNLS-04D1oa & 022511.76 & -041049.4 & 1.006 & 3 \\
\hline SNLS-P04D1bo & SNLS-04D1or & 022623.42 & -044150.7 & 1.139 & 4 \\
\hline SNLS-P04D1bq & SNLS-04D1oe & 022524.50 & -045334.7 & 0.641 & 4 \\
\hline SNLS-P04D1br & SNLS-04D1ln & 022553.45 & -042702.9 & 0.208 & 5 \\
\hline SNLS-P04D1bs & SNLS-04D1kw & 022509.96 & -041513.9 & 0.901 & 3 \\
\hline SNLS-P04D1bv & SNLS-04D1qw & 022625.60 & -043724.6 & 0.312 & 5 \\
\hline SNLS-P04D1bw & SNLS-04D1 ot & 022622.42 & -043113.1 & 1.014 & 3 \\
\hline SNLS-P04D1bx & SNLS-04D1nz & 022515.65 & -044101.3 & 0.264 & 5 \\
\hline SNLS-P04D1by & SNLS-04D1hi & 022621.60 & -041652.7 & 0.556 & 4 \\
\hline SNLS-P04D1ce & $\ldots$ & 022544.81 & -042405.4 & 0.910 & 3 \\
\hline SNLS-P04D1ch & SNLS-04D1qa & 022514.88 & -044922.9 & 0.173 & 5 \\
\hline SNLS-P04D1cj & SNLS-04D1po & 022524.55 & -042109.0 & 0.141 & 5 \\
\hline SNLS-P04D1cm & SNLS-04D1oq & 022439.56 & -041556.6 & 0.330 & 5 \\
\hline SNLS-P04D1cq & $\ldots$ & 022627.24 & -043353.8 & 0.795 & 4 \\
\hline SNLS-P04D1ct & SNLS-04D1pq & 022452.84 & -040740.8 & 1.017 & 4 \\
\hline SNLS-P04D1cu & & 022551.16 & -045917.6 & 1.001 & 3 \\
\hline SNLS-P04D1cy & SNLS-04D1pv & 022432.48 & -042419.9 & 0.873 & 3 \\
\hline SNLS-P04D1cz & SNLS-04D1qs & 022544.81 & -042405.4 & 0.910 & 3 \\
\hline SNLS-P04D1dc & SNLS-04D1qu & 022618.65 & -040438.1 & 0.343 & 4 \\
\hline SNLS-P04D1de & SNLS-04D1qn & 022728.18 & -042037.1 & 0.490 & 3 \\
\hline SNLS-P04D1df & SNLS-04D1pj & 022650.27 & -041208.2 & 0.156 & 5 \\
\hline SNLS-P04D1dg & SNLS-04D1pu & 022728.43 & -044441.5 & 0.639 & 3 \\
\hline SNLS-P04D1dk & SNLS-04D1pf & 022513.69 & -045749.2 & 0.311 & 5 \\
\hline SNLS-P04D1dm & $\ldots$ & 022410.34 & -043815.3 & 0.647 & 4 \\
\hline SNLS-P04D1dn & $\ldots$ & 022420.81 & -043035.7 & 0.640 & 4 \\
\hline SNLS-P04D1dq & SNLS-04D1qr & 022549.10 & -042900.2 & 0.429 & 4 \\
\hline SNLS-P04D1dw & SNLS-04D1sw & 022644.52 & -041639.0 & 0.710 & 4 \\
\hline SNLS-P04D1dx & SNLS-04D1tb & 022409.59 & -045011.8 & 0.495 & 4 \\
\hline SNLS-P04D1dz & SNLS-04D1rg & 022643.64 & -043317.8 & 0.309 & 5 \\
\hline SNLS-P04D1eg & SNLS-05D1ad & 022454.23 & -044347.6 & 0.408 & 5 \\
\hline SNLS-P04D1eh & SNLS-04D1sf & 022556.14 & -043044.7 & 0.707 & 3 \\
\hline SNLS-P04D1ej & SNLS-04D1s1 & 022420.96 & -040350.1 & 0.703 & 3 \\
\hline SNLS-P04D1el & SNLS-04D1sc & 022634.39 & -040246.3 & 0.627 & 4 \\
\hline SNLS-P04D4aa & SNLS-04D4ih & 221717.01 & -174038.9 & 0.935 & 3 \\
\hline SNLS-P04D4aa & SNLS-04D4lj & 221501.49 & -174919.9 & 0.411 & 4 \\
\hline SNLS-P04D4ab & $\ldots$ & 221530.47 & -172315.2 & 1.046 & 3 \\
\hline SNLS-P04D4ab & SNLS-04D4ay & 221554.07 & -180250.1 & 0.565 & 5 \\
\hline SNLS-P04D4ab & SNLS-04D4id & 221621.50 & -171344.2 & 0.770 & 4 \\
\hline SNLS-P04D4ac & $\ldots$ & 221726.04 & -180144.9 & 0.715 & 5 \\
\hline SNLS-P04D4ac & SNLS-04D4kd & 221344.03 & -175758.9 & 0.745 & 5 \\
\hline SNLS-P04D4ad & SNLS-04D4lb & 221459.53 & -180146.1 & 0.800 & 4 \\
\hline SNLS-P04D4ae & SNLS-04D4mt & 221654.10 & -173156.0 & 0.371 & 5 \\
\hline SNLS-P04D4af & SNLS-04D4di & 221540.78 & -174012.7 & 0.947 & 3 \\
\hline SNLS-P04D4ag & SNLS-04D4it & 221600.53 & -180548.5 & 0.982 & 3 \\
\hline SNLS-P04D4ag & SNLS-04D4ms & 221613.40 & -175319.7 & 0.568 & 4 \\
\hline SNLS-P04D4ah & $\ldots$ & 221513.70 & -180319.8 & 0.468 & 4 \\
\hline SNLS-P04D4ah & SNLS-04D4cm & 221328.78 & -180340.6 & 0.466 & 5 \\
\hline SNLS-P04D4ai & $\ldots$ & 221356.80 & -180102.4 & 0.741 & 4 \\
\hline SNLS-P04D4ai & $\ldots$ & 221647.69 & -175947.9 & 0.416 & 5 \\
\hline SNLS-P04D4aj & SNLS-04D4ev & 221510.50 & -180558.1 & 0.900 & 3 \\
\hline SNLS-P04D4ak & SNLS-04D4fu & 221411.42 & -173232.3 & 0.134 & 5 \\
\hline SNLS-P04D4al & SNLS-04D4dw & 221644.63 & -175002.4 & 1.032 & 3 \\
\hline SNLS-P04D4ao & SNLS-04D4im & 221500.83 & -172345.9 & 0.752 & 4 \\
\hline SNLS-P04D4ap & SNLS-04D4ec & 221629.30 & -181104.4 & 0.593 & 5 \\
\hline SNLS-P04D4ap & SNLS-04D4in & 221508.58 & -171540.2 & 0.516 & 4 \\
\hline SNLS-P04D4aq & $\ldots$ & 221509.36 & -181104.5 & 0.886 & 3 \\
\hline SNLS-P04D4aq & SNLS-04D4jw & 221718.88 & -173956.0 & 0.961 & 4 \\
\hline SNLS-P04D4ar & $\ldots$ & 221627.32 & -181218.9 & 0.453 & 5 \\
\hline SNLS-P04D4as & SNLS-04D4eq & 221423.63 & -175204.6 & 0.701 & 5 \\
\hline SNLS-P04D4as & SNLS-04D4jv & 221536.94 & -180924.3 & 0.230 & 5 \\
\hline
\end{tabular}


Table 5. Continued.

\begin{tabular}{|c|c|c|c|c|c|}
\hline Name & RTD Name & RA (J2000) & Dec. (J2000) & Redshift & Quality Flag \\
\hline SNLS-P04D4at & & 221444.87 & -180920.0 & 1.171 & 4 \\
\hline SNLS-P04D4at & SNLS-04D4kq & 221331.67 & -175347.2 & 0.744 & 3 \\
\hline SNLS-P04D4au & SNLS-04D4kb & 221716.25 & -173837.8 & 0.967 & 3 \\
\hline SNLS-P04D4av & SNLS-04D4jx & 221652.10 & -173806.9 & 0.373 & 5 \\
\hline SNLS-P04D4aw & SNLS-04D4lg & 221508.10 & -173941.2 & 0.912 & 4 \\
\hline SNLS-P04D4ax & & 221418.85 & -173232.9 & 1.188 & 3 \\
\hline SNLS-P04D4ax & SNLS-04D4dm & 221525.47 & -171442.9 & 0.812 & 4 \\
\hline SNLS-P04D4ba & & 221609.14 & -180630.8 & 0.933 & 3 \\
\hline SNLS-P04D4bd & SNLS-04D4et & 221451.77 & -174723.0 & 0.536 & 5 \\
\hline SNLS-P04D4bd & SNLS-04D4kn & 221504.42 & -171945.6 & 0.910 & 4 \\
\hline SNLS-P04D4be & SNLS-04D4ew & 221459.83 & -174035.1 & 0.131 & 5 \\
\hline SNLS-P04D4be & SNLS-04D4jn & 221427.43 & -175812.6 & 0.330 & 5 \\
\hline SNLS-P04D4bf & SNLS-04D4eu & 221447.34 & -174611.6 & 0.795 & 3 \\
\hline SNLS-P04D4bh & SNLS-04D4ju & 221702.72 & -171958.7 & 0.472 & 5 \\
\hline SNLS-P04D4bi & SNLS-04D4fo & 221447.10 & -172516.1 & 0.880 & 3 \\
\hline SNLS-P04D4bk & SNLS-04D4fv & 221604.73 & -175931.9 & 0.533 & 4 \\
\hline SNLS-P04D4bk & SNLS-04D4ky & 221727.99 & -181337.1 & 0.559 & 3 \\
\hline SNLS-P04D4b1 & & 221545.53 & -173214.3 & 0.918 & 3 \\
\hline SNLS-P04D4bl & SNLS-04D4mx & 221630.26 & -180341.5 & 1.220 & 3 \\
\hline SNLS-P04D4bm & & 221621.79 & -180404.6 & 0.852 & 4 \\
\hline SNLS-P04D4bm & SNLS-04D4lw & 221553.40 & -175258.6 & 0.850 & 3 \\
\hline SNLS-P04D4bo & $\ldots$ & 221658.55 & -175614.9 & 0.740 & 5 \\
\hline SNLS-P04D4bp & $\ldots$ & 221440.64 & -173537.6 & 0.720 & 4 \\
\hline SNLS-P04D4br & SNLS-04D4gd & 221509.38 & -181335.1 & 0.193 & 4 \\
\hline SNLS-P04D4bt & SNLS-04D4hk & 221634.91 & -174942.1 & 0.587 & 4 \\
\hline SNLS-P04D4bv & SNLS-04D4ft & 221431.08 & -174019.0 & 0.268 & 5 \\
\hline SNLS-P04D4bx & SNLS-04D4hg & 221641.90 & -175631.5 & 0.518 & 5 \\
\hline SNLS-P04D4by & $\ldots$ & 221648.93 & -175320.8 & 0.805 & 4 \\
\hline SNLS-P04D4bz & & 221356.35 & -174945.6 & 1.199 & 5 \\
\hline SNLS-P04D4ca & SNLS-04D4gz & 221658.96 & -173718.4 & 0.375 & 5 \\
\hline SNLS-P04D4cb & & 221403.62 & -173757.3 & 1.002 & 3 \\
\hline SNLS-P04D4cc & SNLS-04D4gg & 221609.23 & -171740.1 & 0.424 & 5 \\
\hline SNLS-P04D4cf & & 221652.33 & -180213.8 & 0.804 & 4 \\
\hline SNLS-P04D4ch & SNLS-04D4hu & 221536.21 & -175020.3 & 0.703 & 5 \\
\hline SNLS-P04D4cj & SNLS-04D4ht & 221433.27 & -172131.5 & 0.218 & 5 \\
\hline SNLS-P04D4ck & SNLS-04D4hx & 221340.55 & -172303.5 & 0.543 & 3 \\
\hline SNLS-P04D4cl & SNLS-04D4ib & 221641.70 & -180618.3 & 0.705 & 4 \\
\hline SNLS-P05D1ac & SNLS-04D1ss & 022648.35 & -042750.8 & 0.328 & 5 \\
\hline SNLS-P05D1ac & SNLS-05D1cq & 022519.09 & -044733.7 & 0.309 & 4 \\
\hline SNLS-P05D1ad & $\ldots$ & 022605.11 & -043807.7 & 0.563 & 5 \\
\hline SNLS-P05D1ae & & 022544.29 & -044129.7 & 0.672 & 3 \\
\hline SNLS-P05D1ae & SNLS-05D1cb & 022657.12 & -040703.1 & 0.632 & 4 \\
\hline SNLS-P05D1af & $\ldots$ & 022705.06 & -035937.8 & 1.037 & 4 \\
\hline SNLS-P05D1ai & SNLS-05D1cf & 022755.52 & -045420.3 & 0.497 & 4 \\
\hline SNLS-P05D1al & $\cdots$ & 022609.43 & -042310.3 & 0.855 & 3 \\
\hline SNLS-P05D1aq & . & 022631.05 & -044702.0 & 0.438 & 4 \\
\hline SNLS-P05D1as & SNLS-05D1ds & 022738.14 & -043933.8 & 0.309 & 5 \\
\hline SNLS-P05D1au & SNLS-05D1da & 022624.54 & -042615.2 & 0.426 & 5 \\
\hline SNLS-P05D1av & SNLS-05D1cs & 022716.12 & -041100.1 & 0.918 & 3 \\
\hline SNLS-P05D1ax & $\ldots$ & 022509.49 & -040220.1 & 0.615 & 5 \\
\hline SNLS-P05D1ba & SNLS-05D1cm & 022713.04 & -042718.5 & 0.228 & 5 \\
\hline SNLS-P05D1bc & $\ldots$ & 022606.73 & -040021.9 & 0.960 & 4 \\
\hline SNLS-P05D1bd & SNLS-05D1di & 022508.46 & -041406.7 & 0.629 & 4 \\
\hline SNLS-P05D1be & $\ldots$ & 022538.24 & -041806.3 & 0.329 & 3 \\
\hline SNLS-P05D1bg & $\ldots$ & 022458.03 & -041927.0 & 0.837 & 3 \\
\hline SNLS-P05D1bl & $\ldots$ & 022635.38 & -040044.0 & 0.211 & 4 \\
\hline SNLS-P05D1bn & & 022718.54 & -045146.8 & 0.436 & 5 \\
\hline SNLS-P05D1bo & SNLS-05D1dd & 022643.99 & -045812.8 & 0.331 & 4 \\
\hline SNLS-P05D1bp & $\ldots$ & 022704.62 & -042648.7 & 0.070 & 4 \\
\hline SNLS-P05D1br & & 022628.91 & -040713.9 & 0.884 & 3 \\
\hline SNLS-P05D1bv & SNLS-05D1je & 022510.71 & -045032.8 & 0.310 & 4 \\
\hline SNLS-P05D1bx & $\ldots$ & 022600.64 & -041852.4 & 0.705 & 4 \\
\hline SNLS-P05D1bz & $\ldots$ & 022442.17 & -041304.3 & 0.834 & 3 \\
\hline
\end{tabular}


Table 5. Continued.

\begin{tabular}{|c|c|c|c|c|c|}
\hline Name & RTD Name & RA (J2000) & Dec. (J2000) & Redshift & Quality Flag \\
\hline SNLS-P05D1ca & SNLS-05D1fa & 022604.81 & -041436.9 & 0.794 & 3 \\
\hline SNLS-P05D1cd & SNLS-05D1ej & 022606.32 & -044346.0 & 0.312 & 4 \\
\hline SNLS-P05D1cg & $\ldots$ & 022635.48 & -042644.0 & 0.690 & 4 \\
\hline SNLS-P05D1cj & $\ldots$ & 022546.98 & -041134.2 & 0.659 & 3 \\
\hline SNLS-P05D1co & $\ldots$ & 022659.77 & -043430.3 & 1.175 & 3 \\
\hline SNLS-P05D1cp & SNLS-05D1fs & 022729.33 & -042248.9 & 0.346 & 4 \\
\hline SNLS-P05D1cq & $\ldots$ & 022633.35 & -040038.7 & 0.345 & 4 \\
\hline SNLS-P05D1cu & $\ldots$ & 022415.86 & -043520.5 & 1.182 & 3 \\
\hline SNLS-P05D1cw & $\ldots$ & 022734.29 & -043811.4 & 0.585 & 4 \\
\hline SNLS-P05D1cx & $\ldots$ & 022705.30 & -043746.0 & 0.071 & 4 \\
\hline SNLS-P05D1cz & $\ldots$ & 022707.55 & -042358.5 & 0.237 & 5 \\
\hline SNLS-P05D1da & SNLS-05D1jv & 022628.34 & -042014.4 & 0.422 & 4 \\
\hline SNLS-P05D1dd & $\ldots$ & 022432.80 & -041332.8 & 0.259 & 4 \\
\hline SNLS-P05D1de & $\ldots$ & 022755.92 & -045457.4 & 1.140 & 3 \\
\hline SNLS-P05D1dg & $\ldots$ & 022546.23 & -043129.4 & 1.057 & 3 \\
\hline SNLS-P05D1dh & $\ldots$ & 022628.26 & -040051.7 & 0.209 & 5 \\
\hline SNLS-P05D1di & SNLS-05D1ib & 022534.35 & -045836.7 & 0.238 & 5 \\
\hline SNLS-P05D1dj & $\ldots$ & 022706.66 & -043608.7 & 0.228 & 5 \\
\hline SNLS-P05D1dk & $\ldots$ & 022526.65 & -041844.0 & 0.644 & 4 \\
\hline SNLS-P05D1dl & $\ldots$ & 022746.67 & -045356.1 & 0.294 & 4 \\
\hline SNLS-P05D1dm & $\ldots$ & 022712.69 & -045727.9 & 0.191 & 4 \\
\hline SNLS-P05D1dn & $\ldots$ & 022459.66 & -044756.8 & 0.439 & 4 \\
\hline SNLS-P05D1do & SNLS-05D1hm & 022746.16 & -044302.1 & 0.586 & 4 \\
\hline SNLS-P05D1dp & SNLS-05D1ie & 022423.58 & -044039.4 & 0.263 & 5 \\
\hline SNLS-P05D1dr & $\ldots$ & 022641.52 & -040001.8 & 0.877 & 4 \\
\hline SNLS-P05D1ds & $\ldots$ & 022523.57 & -040510.4 & 0.600 & 4 \\
\hline SNLS-P05D1dt & $\ldots$ & 022729.96 & -044931.9 & 0.695 & 4 \\
\hline SNLS-P05D1du & $\ldots$ & 022657.65 & -045438.6 & 0.141 & 5 \\
\hline SNLS-P05D1dw & $\ldots$ & 022702.23 & -044906.4 & 0.621 & 4 \\
\hline SNLS-P05D1ec & $\ldots$ & 022728.06 & -043848.3 & 0.298 & 4 \\
\hline SNLS-P05D1ed & $\ldots$ & 022716.08 & -043503.7 & 0.357 & 5 \\
\hline SNLS-P05D1ee & $\ldots$ & 022702.49 & -043531.2 & 0.030 & 5 \\
\hline SNLS-P05D1ef & SNLS-05D1jq & 022412.10 & -044141.8 & 0.500 & 5 \\
\hline SNLS-P05D1ei & $\ldots$ & 022716.41 & -042252.3 & 0.514 & 4 \\
\hline SNLS-P05D1em & $\ldots$ & 022653.65 & -040414.1 & 0.683 & 4 \\
\hline SNLS-P05D1en & $\ldots$ & 022539.89 & -040525.0 & 0.063 & 5 \\
\hline SNLS-P05D1eq & SNLS-05D1jh & 022425.48 & -040347.4 & 0.765 & 4 \\
\hline SNLS-P05D1ev & SNLS-05D1ku & 022613.23 & -042414.7 & 0.319 & 5 \\
\hline SNLS-P05D1ey & SNLS-05D1kh & 022556.06 & -041329.4 & 0.327 & 5 \\
\hline SNLS-P05D1ez & $\ldots$ & 022603.81 & -044209.8 & 0.603 & 4 \\
\hline SNLS-P05D1ff & $\ldots$ & 022746.42 & -040825.9 & 0.874 & 4 \\
\hline SNLS-P05D1fi & $\ldots$ & 022455.14 & -045335.2 & 0.710 & 4 \\
\hline SNLS-P05D1fj & $\ldots$ & 022626.00 & -045446.7 & 0.658 & 3 \\
\hline SNLS-P05D1fm & $\ldots$ & 022545.14 & -045523.0 & 0.903 & 3 \\
\hline SNLS-P05D1ft & $\ldots$ & 022400.31 & -040433.2 & 0.571 & 4 \\
\hline SNLS-P05D1fv & $\ldots$ & 022755.05 & -044956.9 & 0.676 & 3 \\
\hline SNLS-P05D1fw & $\ldots$ & 022711.32 & -045020.4 & 0.964 & 4 \\
\hline SNLS-P05D1fx & $\ldots$ & 022715.90 & -044946.3 & 0.722 & 4 \\
\hline SNLS-P05D1gk & $\ldots$ & 022417.30 & -045503.6 & 0.965 & 4 \\
\hline SNLS-P05D1gm & $\ldots$ & 022730.80 & -044013.9 & 0.578 & 5 \\
\hline SNLS-P05D1gn & $\ldots$ & 022718.37 & -043347.9 & 0.309 & 5 \\
\hline SNLS-P05D1gu & $\ldots$ & 022500.92 & -041729.2 & 0.863 & 4 \\
\hline SNLS-P05D1gv & $\ldots$ & 022458.70 & -041738.7 & 0.254 & 4 \\
\hline SNLS-P05D1gw & $\ldots$ & 022446.34 & -041829.5 & 0.669 & 4 \\
\hline SNLS-P05D1gy & $\ldots$ & 022405.31 & -041925.4 & 0.557 & 4 \\
\hline SNLS-P05D1gz & $\ldots$ & 022403.91 & -041804.5 & 0.466 & 5 \\
\hline SNLS-P05D1hb & $\ldots$ & 022732.99 & -040030.0 & 0.706 & 4 \\
\hline SNLS-P05D1hc & $\ldots$ & 022727.07 & -040720.5 & 0.255 & 5 \\
\hline SNLS-P05D1hd & $\ldots$ & 022704.24 & -040517.3 & 0.767 & 3 \\
\hline SNLS-P05D1hf & $\ldots$ & 022522.38 & -040931.9 & 0.558 & 5 \\
\hline SNLS-P05D1hg & $\ldots$ & 022409.98 & -041316.6 & 1.050 & 3 \\
\hline SNLS-P05D1hi & SNLS-05D1ma & 022419.58 & -040804.0 & 0.432 & 4 \\
\hline SNLS-P05D1hk & SNLS-05D1lo & 022554.37 & -042004.7 & 0.862 & 4 \\
\hline
\end{tabular}


Table 5. Continued.

\begin{tabular}{|c|c|c|c|c|c|}
\hline Name & RTD Name & RA (J2000) & Dec. (J2000) & Redshift & Quality Flag \\
\hline SNLS-P05D4aa & SNLS-05D4ff & 221620.16 & -180233.0 & 0.403 & 5 \\
\hline SNLS-P05D4ab & SNLS-05D4ek & 221627.49 & -174409.3 & 0.536 & 4 \\
\hline SNLS-P05D4ac & SNLS-05D4al & 221544.81 & -175159.3 & 0.308 & 5 \\
\hline SNLS-P05D4ac & SNLS-05D4fl & 221501.83 & -175636.3 & 0.308 & 5 \\
\hline SNLS-P05D4ad & SNLS-05D4bc & 221722.47 & -180846.1 & 0.127 & 5 \\
\hline SNLS-P05D4ad & SNLS-05D4fg & 221641.34 & -173544.9 & 0.840 & 4 \\
\hline SNLS-P05D4ae & SNLS-05D4bf & 221626.36 & -181353.6 & 0.553 & 5 \\
\hline SNLS-P05D4af & $\ldots$ & 221656.54 & -175211.5 & 0.745 & 5 \\
\hline SNLS-P05D4af & $\ldots$ & 221704.45 & -181220.9 & 0.618 & 4 \\
\hline SNLS-P05D4ag & & 221653.55 & -175758.2 & 0.740 & 4 \\
\hline SNLS-P05D4ag & SNLS-05D4ay & 221433.16 & -174603.2 & 0.409 & 4 \\
\hline SNLS-P05D4ah & SNLS-05D4be & 221653.39 & -171410.1 & 0.538 & 4 \\
\hline SNLS-P05D4ai & SNLS-05D4bn & 221607.41 & -173040.7 & 0.878 & 4 \\
\hline SNLS-P05D4ai & SNLS-05D4fi & 221609.58 & -171416.2 & 0.411 & 5 \\
\hline SNLS-P05D4aj & & 221440.11 & -173735.2 & 0.657 & 4 \\
\hline SNLS-P05D4ak & SNLS-05D4av & 221410.49 & -175442.6 & 0.508 & 4 \\
\hline SNLS-P05D4al & & 221407.08 & -173921.8 & 0.793 & 3 \\
\hline SNLS-P05D4am & SNLS-05D4bl & 221647.53 & -171644.0 & 1.286 & 3 \\
\hline SNLS-P05D4an & SNLS-05D4bi & 221556.53 & -175909.7 & 0.776 & 3 \\
\hline SNLS-P05D4ao & SNLS-05D4fp & 221432.56 & -172430.0 & 1.014 & 3 \\
\hline SNLS-P05D4ap & & 221647.45 & -181215.8 & 0.973 & 4 \\
\hline SNLS-P05D4aq & $\ldots$ & 221651.11 & -174555.3 & 0.939 & 4 \\
\hline SNLS-P05D4ar & $\ldots$ & 221537.70 & -171410.9 & 0.923 & 4 \\
\hline SNLS-P05D4ar & SNLS-05D4ar & 221430.52 & -180219.6 & 0.191 & 5 \\
\hline SNLS-P05D4as & $\ldots$ & 221435.03 & -172515.9 & 0.479 & 5 \\
\hline SNLS-P05D4at & $\ldots$ & 221702.83 & -172131.6 & 1.030 & 3 \\
\hline SNLS-P05D4at & SNLS-05D4jq & 221450.86 & -173323.3 & 1.081 & 4 \\
\hline SNLS-P05D4au & ... & 221447.72 & -173619.2 & 1.187 & 3 \\
\hline SNLS-P05D4aw & $\ldots$ & 221523.09 & -181357.6 & 0.685 & 3 \\
\hline SNLS-P05D4ax & & 221614.60 & -180900.3 & 0.840 & 4 \\
\hline SNLS-P05D4ax & SNLS-05D4jp & 221344.29 & -174955.2 & 0.883 & 4 \\
\hline SNLS-P05D4ay & SNLS-05D4bw & 221431.39 & -180131.5 & 0.259 & 5 \\
\hline SNLS-P05D4ay & SNLS-05D4gx & 221355.94 & -173204.0 & 0.300 & 5 \\
\hline SNLS-P05D4az & & 221328.98 & -175249.1 & 0.792 & 4 \\
\hline SNLS-P05D4ba & SNLS-05D4bm & 221704.60 & -174039.9 & 0.373 & 5 \\
\hline SNLS-P05D4bb & & 221433.00 & -171738.2 & 0.420 & 5 \\
\hline SNLS-P05D4bc & SNLS-05D4ck & 221601.52 & -180459.2 & 0.459 & 5 \\
\hline SNLS-P05D4bd & SNLS-05D4ch & 221413.84 & -172242.4 & 0.856 & 3 \\
\hline SNLS-P05D4be & & 221357.64 & -181236.6 & 0.397 & 4 \\
\hline SNLS-P05D4be & SNLS-05D4jz & 221605.43 & -174216.0 & 0.516 & 4 \\
\hline SNLS-P05D4bg & SNLS-05D4cw & 221450.05 & -174419.9 & 0.376 & 4 \\
\hline SNLS-P05D4bg & SNLS-05D4gy & 221628.50 & -175149.4 & 0.588 & 4 \\
\hline SNLS-P05D4bh & SNLS-05D4jy & 221622.93 & -174659.4 & 0.868 & 4 \\
\hline SNLS-P05D4bi & SNLS-05D4de & 221546.38 & -172749.8 & 0.644 & 4 \\
\hline SNLS-P05D4bi & SNLS-05D4lb & 221354.16 & -172440.2 & 0.797 & 4 \\
\hline SNLS-P05D4bj & SNLS-05D4cb & 221557.36 & -175418.8 & 0.208 & 5 \\
\hline SNLS-P05D4bj & SNLS-05D4kp & 221431.44 & -175750.4 & 0.409 & 5 \\
\hline SNLS-P05D4bk & SNLS-05D4ca & 221411.32 & -174815.2 & 0.607 & 5 \\
\hline SNLS-P05D4bk & SNLS-05D4is & 221708.06 & -180130.6 & 0.837 & 3 \\
\hline SNLS-P05D4bl & SNLS-05D4cn & 221331.43 & -171720.8 & 0.764 & 4 \\
\hline SNLS-P05D4bl & SNLS-05D4jw & 221714.21 & -175508.5 & 0.309 & 4 \\
\hline SNLS-P05D4bm & SNLS-05D4cq & 221409.68 & -181334.6 & 0.701 & 4 \\
\hline SNLS-P05D4bm & SNLS-05D4jx & 221455.55 & -173023.2 & 0.811 & 4 \\
\hline SNLS-P05D4bn & SNLS-05D4dd & 221617.23 & -172231.5 & 0.791 & 4 \\
\hline SNLS-P05D4bo & $\ldots$ & 221357.28 & -180247.7 & 0.590 & 4 \\
\hline SNLS-P05D4br & SNLS-05D4dt & 221425.85 & -174015.9 & 0.407 & 5 \\
\hline SNLS-P05D4bs & SNLS-05D4dr & 221551.17 & -180610.4 & 1.033 & 3 \\
\hline SNLS-P05D4bt & & 221610.33 & -175531.7 & 0.441 & 5 \\
\hline SNLS-P05D4bu & SNLS-05D4dp & 221555.41 & -172906.1 & 0.340 & 5 \\
\hline SNLS-P05D4bx & & 221349.29 & -172527.7 & 0.952 & 3 \\
\hline SNLS-P05D4by & SNLS-05D4dn & 221601.37 & -175717.3 & 0.191 & 5 \\
\hline SNLS-P05D4bz & SNLS-05D4dx & 221339.37 & -180321.0 & 0.792 & 4 \\
\hline SNLS-P05D4ca & SNLS-05D4eq & 221407.74 & -175235.8 & 0.950 & 3 \\
\hline
\end{tabular}


Table 5. Continued.

\begin{tabular}{|c|c|c|c|c|c|}
\hline Name & RTD Name & RA (J2000) & Dec. (J2000) & Redshift & Quality Flag \\
\hline SNLS-P05D4cb & SNLS-05D4en & 221413.79 & -171660.0 & 0.422 & 5 \\
\hline SNLS-P05D4cc & SNLS-05D4du & 221529.58 & -175405.4 & 0.306 & 5 \\
\hline SNLS-P05D4cd & SNLS-05D4fc & 221653.04 & -174113.4 & 1.065 & 3 \\
\hline SNLS-P05D4ce & $\ldots$ & 221507.24 & -171658.5 & 0.969 & 3 \\
\hline SNLS-P05D4ch & $\ldots$ & 221537.65 & -180421.7 & 1.171 & 4 \\
\hline SNLS-P05D4ci & SNLS-05D4er & 221604.12 & -173400.3 & 1.069 & 4 \\
\hline SNLS-P05D4cl & SNLS-05D4ej & 221552.51 & -181144.3 & 0.586 & 5 \\
\hline SNLS-P06D1ac & SNLS-06D1ac & 022407.83 & -045552.7 & 0.416 & 4 \\
\hline SNLS-P06D1ag & & 022421.65 & -044008.5 & 0.641 & 5 \\
\hline SNLS-P06D1ah & SNLS-05D1lz & 022527.53 & -042842.8 & 0.461 & 5 \\
\hline SNLS-P06D1ai & $\ldots$ & 022514.10 & -041659.8 & 0.863 & 3 \\
\hline SNLS-P06D1aj & SNLS-05D1ln & 022445.17 & -042037.2 & 0.656 & 4 \\
\hline SNLS-P06D1am & SNLS-05D1lj & 022552.16 & -040940.2 & 0.956 & 3 \\
\hline SNLS-P06D4aa & $\ldots$ & 221621.09 & -172754.1 & 0.555 & 5 \\
\hline SNLS-P06D4ac & SNLS-06D4bw & 221503.71 & -175260.0 & 0.732 & 5 \\
\hline SNLS-P06D4ad & $\ldots$ & 221510.87 & -173229.1 & 0.556 & 3 \\
\hline SNLS-P06D4af & $\ldots$ & 221337.14 & -173545.5 & 0.991 & 3 \\
\hline SNLS-P06D4ag & $\ldots$ & 221656.62 & -172534.0 & 1.267 & 3 \\
\hline SNLS-P06D4ah & SNLS-06D4bz & 221344.15 & -171720.5 & 0.663 & 4 \\
\hline SNLS-P06D4ai & SNLS-06D4cd & 221639.20 & -180003.8 & 0.590 & 5 \\
\hline SNLS-P06D4an & SNLS-06D4bo & 221528.05 & -172434.0 & 0.552 & 4 \\
\hline SNLS-P06D4ao & SNLS-06D4cb & $22 \quad 1516.34$ & -173700.8 & 0.440 & 5 \\
\hline SNLS-P06D4ar & SNLS-06D4dp & 221435.23 & -171738.8 & 1.126 & 4 \\
\hline SNLS-P06D4as & $\ldots$ & 221734.79 & -175655.1 & 0.840 & 4 \\
\hline SNLS-P06D4av & $\ldots$ & 221543.58 & -181048.3 & 0.561 & 4 \\
\hline SNLS-P06D4aw & SNLS-06D4co & 221526.49 & -175209.0 & 0.439 & 4 \\
\hline SNLS-P06D4ax & SNLS-06D4dl & 221659.80 & -171502.9 & 0.753 & 4 \\
\hline SNLS-P06D4ay & $\ldots$ & 221624.10 & -175044.7 & 1.161 & 3 \\
\hline SNLS-P06D4az & SNLS-06D4du & 221547.37 & -175441.9 & 0.602 & 4 \\
\hline SNLS-P06D4ba & $\ldots$ & 221555.56 & -174136.3 & 0.665 & 4 \\
\hline SNLS-P06D4bb & SNLS-06D4cm & 221515.75 & -171811.8 & 0.918 & 3 \\
\hline SNLS-P06D4bc & SNLS-06D4ds & 221601.40 & -180520.7 & 0.318 & 5 \\
\hline SNLS-P06D4bd & SNLS-06D4cq & 221655.48 & -174243.4 & 0.413 & 5 \\
\hline SNLS-P06D4be & SNLS-06D4dt & 221405.87 & -173539.7 & 1.105 & 3 \\
\hline SNLS-P06D4bf & $\ldots$ & 221535.59 & -180108.7 & 0.369 & 5 \\
\hline SNLS-P06D4bj & SNLS-06D4eb & 221545.04 & -180424.9 & 0.652 & 4 \\
\hline SNLS-P06D4bk & SNLS-06D4dv & 221531.90 & -181033.6 & 0.129 & 5 \\
\hline SNLS-P06D4bm & SNLS-06D4dh & 221419.57 & -173505.2 & 0.304 & 5 \\
\hline SNLS-P06D4bo & SNLS-06D4do & 221548.60 & -171700.7 & 0.290 & 5 \\
\hline SNLS-P06D4br & SNLS-06D4el & 221627.25 & -175122.7 & 0.587 & 4 \\
\hline SNLS-P06D4bs & $\ldots$ & 221459.91 & -173137.1 & 1.166 & 3 \\
\hline SNLS-P06D4bt & $\ldots$ & 221635.03 & -172545.7 & 0.784 & 4 \\
\hline SNLS-P06D4bv & $\ldots$ & 221433.12 & -171954.8 & 0.752 & 4 \\
\hline SNLS-P06D4bw & SNLS-06D4ee & 221329.35 & -175547.6 & 0.651 & 4 \\
\hline SNLS-P06D4bx & & 221410.66 & -180844.2 & 1.111 & 3 \\
\hline SNLS-P06D4bz & SNLS-06D4gq & 221424.34 & -172406.6 & 0.990 & 4 \\
\hline SNLS-P06D4ca & $\ldots$ & 221616.62 & -180254.7 & 0.784 & 4 \\
\hline SNLS-P06D4cb & $\ldots$ & 221554.01 & -174408.7 & 0.999 & 4 \\
\hline SNLS-P06D4cd & SNLS-06D4fo & 221349.45 & -173626.2 & 0.806 & 4 \\
\hline SNLS-P06D4ce & $\ldots$ & 221713.68 & -172224.9 & 0.969 & 3 \\
\hline SNLS-P06D4cf & SNLS-06D4fa & 221339.66 & -172414.6 & 0.720 & 4 \\
\hline SNLS-P06D4ch & SNLS-06D4fc & 221351.06 & -171931.3 & 0.678 & 4 \\
\hline SNLS-P06D4ci & SNLS-06D4hh & 221552.04 & -181350.5 & 0.599 & 5 \\
\hline SNLS-P06D4cj & & 221328.59 & -181157.3 & 0.187 & 5 \\
\hline SNLS-P06D4ck & SNLS-06D4gp & 221553.26 & -174729.8 & 0.681 & 4 \\
\hline
\end{tabular}

\section{REFERENCES}

Adami, C. 2011, A\&A, 526, A18

Allende Prieto, C., Hubeny, I., \& Smith, J. A. 2009, MNRAS, 396, 759
Astier, P., et al. 2006, A\&A, 447, 31

Balland, C., et al. 2009, A\&A, 507, 85

Bazin, G., et al. 2009, A\&A, 499, 653

Bazin, G., et al. 2011, A\&A, 534, A43 
Bernstein, J. P., et al. 2012, Apj, 753, 152

Bohlin, R. C. 2000, AJ, 120, 437

Bohlin, R. C. 2007, in ASP Conf. Ser. Vol. 364, The Future of Photometric, Spectrophotometric and Polarimetric Standardization, ed. C. Sterken (San Francisco, CA: ASP), 315

Boulade, O., et al. 2003, SPIE, 4841, 72

Bronder, T. J., et al. 2008, A\&A, 477, 717

Carlberg, R. G., et al. 2008, ApJ, 682, L25

Conley, A., et al. 2011, ApJS, 192, 1

Dawson, K. S., et al. 2009, AJ, 138, 1271

Drinkwater, M. J., et al. 2010, MNRAS, 401, 1429

Ellis, R. S., et al. 2008, ApJ, 674, 51

Fan, X. 1999, AJ, 117, 2528

Flaugher, B. L., et al. 2010, SPIE, Vol. 7735, 77350D

Guy, J., et al. 2010, A\&A, 523, A7

Hamuy, M., et al. 1995, AJ, 109, 1

Hamuy, M., et al. 2000, AJ, 120, 1479

Howell, D. A., et al. 2005, ApJ, 634, 1190
Ilbert, O., et al. 2006, A\&A, 457, 841

Keller, S. C., et al. 2007, PASA, 24, 1

Limboz, F., Karataş, Y., Kilic, M., Benoist, C., \& Aliş, S. 2008, MNRAS, 383, 957

Miszalski, B., Shortridge, K., Saunders, W., Parker, Q. A., \& Croom, S. M. 2006, MNRAS, 371, 1537

Perlmutter, S., et al. 1999, ApJ, 517, 565

Perrett, K., et al. 2010, AJ, 140, 518

Pierre, M., et al. 2006, MNRAS, 372, 591

Pierre, M., et al. 2007, MNRAS, 382, 279

Riess, A. G., et al. 1998, AJ, 116, 1009

Smith, G. A., et al. 2004, SPIE, 5492, 410

Sullivan, M., et al. 2006a, AJ, 131, 960

Sullivan, M., et al. 2006b, ApJ, 648, 868

Sullivan, M., et al. 2010, MNRAS, 406, 782

Sullivan, M., et al. 2011, ApJ, 737, 102

Suzuki, N., et al. 2011, ArXiv e-prints

Walker, E. S., et al. 2011, MNRAS, 410, 1262 